\title{
Absence of coreshine in the Gum/Vela region ${ }^{\star}$ (Research Note)
}

\author{
L. Pagani ${ }^{1}$, C. Lefèvre ${ }^{1}$, A. Bacmann ${ }^{2}$, and J. Steinacker ${ }^{2,3}$ \\ 1 LERMA \& UMR 8112 du CNRS, Observatoire de Paris, 61 Av. de l'Observatoire, 75014 Paris, France \\ e-mail: laurent.pagani@obspm.fr \\ 2 UJF-Grenoble 1/CNRS-INSU, Institut de Plantologie et d'Astrophysique de Grenoble (IPAG) UMR 5274, 38041 Grenoble, France \\ 3 Max-Planck-Institut für Astronomie, Königstuhl 17, 69117 Heidelberg, Germany
}

Received 16 February 2012 / Accepted 8 April 2012

\section{ABSTRACT}

\begin{abstract}
Context. We recently discovered mid-infrared light scattering by micron-size grains deeply buried in dark clouds. We have named this coreshine. We also showed that this effect is widespread across the Galaxy except in the Gum/Vela region, the only region among those we explored without any trace of coreshine.

Aims. We aim to check whether the Gum/Vela situation is a chance effect or if coreshine is really absent from the region.

Methods. We explored the entire available Spitzer/InfraRed Red Array Camera (IRAC) archive centered on the Gum/Vela region in search of the coreshine effect.

Results. Out of 24 validated objects (of a total of 32), we found three cases of coreshine and three possible other cases, while we detect nine cases of non-coreshine emission (bright rimmed clouds - BRC - or polycyclic aromatic hydrocarbon - PAH - emission). This is markedly different from our previous galactic-wide survey with a ratio of $7-8$ coreshine cases per PAH case. In Gum/Vela, a majority of the clouds with protostars or young stellar objects do not show a coreshine effect, while in the galactic-wide survey, $75 \%$ of the protostellar clouds do.

Conclusions. The rare occurence of coreshine, outnumbered by PAH and BRC cases, together with a large number of protostars, let us conclude that the Gum Nebula is a supernova remnant (SNR), and that the blast wave has both reset the grain size distribution and induced the formation of several protostars. The absence of coreshine in the vicinity of several of the Class I objects also implies that the growth time for grains to efficiently scatter mid-infrared radiation exceeds the Class I life duration, which is typically $2 \times 10^{5}$ years, and it also implies that the blast wave has reached these clouds only recently despite the age of the Gum region (over $1.5 \mathrm{My}$ ). This is consistent with their large distance from the center of the SNR.
\end{abstract}

Key words. ISM: supernova remnants - dust, extinction - ISM: structure - ISM: clouds - ISM: individual objects: Gum ISM: individual objects: Vela

\section{Introduction}

\subsection{Supernovae and dust grains}

Supernovae have a profound impact on their surroundings and especially the remnant of the native cloud where their progenitor star formed. Because they are produced by massive stars at the end of their life, these stars have too short a life (a few million or tens of million years) to be able to significantly run away from their parental cloud when they die (though they have already destroyed a sizeable fraction of the cloud during their HII region period). Among other effects on their surroundings, supernovae heavily reprocess dust, whether dominated by destruction of the large grains when the shock wave is not too rapid (shattering for $V_{\text {shock }}<20 \mathrm{~km} \mathrm{~s}^{-1}$, vaporisation between 20 and $80 \mathrm{~km} \mathrm{~s}^{-1}$ ) or by erosion of the small grains when the shock wave is fast or very fast $\left(>50 \mathrm{~km} \mathrm{~s}^{-1}\right.$ or more, with a distinction between thermal sputtering and non-thermal sputtering as a function of velocity) as discussed in a series of papers by Jones et al. (1994, 1996) and Guillet et al. (2007, 2009, 2011). In contrast, Borkowski \& Dwek (1995) found that the velocity threshold between shatteringdominated and sputtering-dominated grain destruction would instead be at about $200 \mathrm{~km} \mathrm{~s}^{-1}$. Recently, Micelotta et al. (2010)

\footnotetext{
* Appendices $\mathrm{B}$ and $\mathrm{C}$ are available in electronic form at http://www. aanda.org
}

have shown that small polycyclic aromatic hydrocarbons (PAHs) would not survive shock velocities above $100 \mathrm{~km} \mathrm{~s}^{-1}$, and all PAHs should be completely destroyed in shocks with velocities above $125 \mathrm{~km} \mathrm{~s}^{-1}$. On the observational side, progress has been made thanks to the Infrared Space Observatory (ISO) and more recently to the Spitzer Space Telescope. These infrared observatories allow one to carry out detailed studies of dust in shock regions, especially near supernova remnants (SNR). While Sankrit et al. (2010) and Arendt et al. (2010) reported on dust destruction by fast shocks (sputtering) in individual cases (Cygnus Loop SNR and Puppis SNR, respectively, with a reported shock velocity of 350 and $500 \mathrm{~km} \mathrm{~s}^{-1}$, respectively), Andersen et al. (2011) analyzed 14 young SNRs and found that in most cases, the nearby clouds have seen their grains being shattered from big grains (BG) to very small grain (VSG). The VSG/BG ratio is found to be two to three times higher in the vicinity of the SNRs than in the plane of the Milky Way and the associated shock velocity is entirely in the $50-80 \mathrm{~km} \mathrm{~s}^{-1}$ range for these sources. On the other hand, the VSG/BG ratio is lower for two of their SNRs (G11.2-0.3, G344.7-0.1) than in the Milky Way plane, suggesting a lack of VSG and PAHs owing to efficient sputtering, similar to the Puppis and Cygnus Loop cases. Andersen et al. (2011) indicate a velocity of $280 \mathrm{~km} \mathrm{~s}^{-1}$, consistent with the sputtering diagnostic for these two SNRs. All these SNRs are relatively young, less than 10000 years. 


\subsection{Absence of coreshine in the Gum/Vela region}

Coreshine, an effect we discovered recently (Steinacker et al. 2010), is starlight in the $3-5 \mu \mathrm{m}$ wavelength range efficiently scattered by grown grains ( 0.5 to $1 \mu \mathrm{m}$ in radius) and is seen toward deeply buried cloud cores. It is characterized by a combination of emission at 3-5 $\mu \mathrm{m}$ and absorption at $8-12 \mu \mathrm{m}$. In a systematic survey of star-forming regions to search for the coreshine effect (Pagani et al. 2010), we noticed that apart from the Galactic plane itself, which is too crowded with stars to see the effect, only one region was completely devoid of clouds showing coreshine. This region, the Gum/Vela region is often, but not always, considered to be a wide supernova remnant, and we suggested that the presence of one or several supernovae could be the reason for this lack of coreshine. For slow shocks, big grains are expected to be shattered and therefore the Gum/Vela supernovae could have destroyed the big grains in this region, suppressing the coreshine effect. Owing to its old age (at least $1 \mathrm{My}$ ), the question arises why the coreshine effect has not reappeared yet, a question that cannot be asked for the other SNRs, which are too young to have had the chance to rebuild big grains. Does this set a lower limit to the time grains take to build up? Though the physics behind the coreshine effect is not yet understood beyond the mere fact that it needs big grains to be effective, it is interesting to study this SNR in more detail to bring some constraints on the mechanism that makes the grains big. Another problem is that in the Galactic-wide survey, only six objects belonging to this region were checked and the statistics are too limited and need to be expanded. We have therefore explored all available data around the Gum/Vela region in the Spitzer IRAC archive to check for the existence of coreshine effect in each individual cloud.

The paper is organized as follows: Sect. 2 describes the Gum/Vela region, Sect. 3 briefly presents the data we have collected from the archive and their analysis, and Sect. 4 presents the discussion on the structure of the region and the meaning of the quasi-absence of coreshine. Appendix A discusses some statistics of the Pagani et al. (2010) paper that are useful for the present data analysis. Appendix B presents the images of all objects discussed here, and Appendix $\mathrm{C}$ presents zoomed images of the cases for which coreshine is visible or probable.

\section{The Gum/Vela region}

The Gum nebula is centered toward the Vela constellation and its diameter is larger than $30^{\circ}$. The region was first discovered through its $\mathrm{H} \alpha$ emission in 1952 (Gum 1952), but it took 20 years before its actual size in $\mathrm{H} \alpha$ emission was correctly estimated (Beuermann 1973; Chanot \& Sivan 1983; Sahu \& Sahu 1993, for a summary). The nebula is also visible via its Hi $21 \mathrm{~cm}$ emission but with markedly different shape and size (Reynoso \& Dubner 1997). Even at small scale the two images show little correlation. Gum (1952) originally suggested that this was a large HII region ionized by $\zeta$ Puppis and $\gamma^{2}$ Velorum. Beuermann (1973) backed up this hypothesis, suggesting also that the stellar wind from these two stars could have created the observed cavity. In the meanwhile, Brandt et al. (1971) showed that the UV flux from these two stars would not be sufficient to sustain the nebula ionization and concluded that a supernova was necessary, possibly the Vela X supernova. Reynolds (1976a,b) claimed that the UV flux was sufficient but most importantly found that most of the emission comes from a region of $250 \mathrm{pc}$ in diameter with an expansion velocity of $20 \mathrm{~km} \mathrm{~s}^{-1}$. This result has backed up several claims that the region was an SNR. Though some authors found no trace of expansion from $\mathrm{H} \alpha$ emission line measurements (Hippelein \& Weinberger 1975) or from Ca II and $\mathrm{Na}$ I absorption line measurements (Wallerstein et al. 1980), more recent studies (Srinivasan et al. 1987; Sahu \& Sahu 1993; Woermann et al. 2001) converged to show that a slow expansion $\left(\sim 10 \mathrm{~km} \mathrm{~s}^{-1}\right)$ does exist. Though the SNR status of the region seems generally accepted, but not by all authors (Choudhury \& Bhatt 2009; Urquhart et al. 2009, e.g., treat this region as a pure HII region), several problems remain. The position and distance to the Sun of the SNR center are debated. They depend on whether the $\mathrm{H} \alpha$ emission (which differs from the Hi emission region) traces the SNR or not (Leahy et al. 1992; Yamaguchi et al. 1999; Woermann et al. 2001; Sushch et al. 2011). The distance to the SNR center varies from 300 to 800 pc (Franco 1990; Sahu 1992), with a majority of values between 400 and 500 pc (e.g. Brandt et al. 1971; Sridharan 1992; Rajagopal \& Srinivasan 1998; Yamaguchi et al. 1999; Woermann et al. 2001). The Gum nebula is not symmetrical, which may be due to an inhomogeneous interstellar medium (ISM) in which the expansion velocity would vary and/or to multiple supernovae as Tenorio-Tagle \& Bodenheimer (1988) have proposed. Gum (1952) indicated that the $\mathrm{H} \alpha$ emission center is $\left(l=258^{\circ}, b=-2^{\circ}\right)$, a value that has been confirmed by Chanot \& Sivan (1983), while the Hr $21 \mathrm{~cm}$ emission center is displaced by $4^{\circ}\left(l=262^{\circ}, b=-3^{\circ}\right.$, Reynoso $\&$ Dubner 1997). A third center has been derived from the intersection of the directions pointed at by the numerous cometary globules (CG) observed in the region $\left(l=264^{\circ}, b=-4^{\circ}\right.$, Sridharan 1992). Woermann et al. (2001) summarized previous work on the cometary globules center of expansion determination and proposed to take an average of these values, that is $\left(l=260.8^{\circ}, b=-5.9^{\circ}\right)$. They suggested that the cometary globules are linked to a supernova explosion that occured $1.5 \mathrm{My}$ ago and that the binary companion of $\zeta$ Puppis could be the progenitor of the supernova. These results were contested by Choudhury \& Bhatt (2009), however. Woermann et al. (2001) also proposed that the center of the expanding shell is $l=261^{\circ}, b=-2.5^{\circ}$, which is within $1 \sigma$ of the cometary globules center of expansion, that the shell is asymmetrical with the front-face shell wall at $130 \mathrm{pc}$ from the center with an expansion velocity of $14 \mathrm{~km} \mathrm{~s}^{-1}$, while the back-face would be only 70 pc deep with an expansion velocity of $8.5 \mathrm{~km} \mathrm{~s}^{-1}$. These values suppose a center of expansion at $500 \mathrm{pc}$ from the sun, but a slightly different fit with a center at $400 \mathrm{pc}$ would also work. Following Yamaguchi et al. (1999), we selected as center position $\alpha_{\mathrm{J} 2000}=08^{\mathrm{h}} 22^{\mathrm{m}} 53.6^{\mathrm{s}}$, $\delta_{\mathrm{J} 2000}=-42^{\circ} 25^{\prime} 20^{\prime \prime}$ and a distance to the Sun of $450 \mathrm{pc}$. In front of the Gum nebula sits the Vela complex (Vela SNR, Iras Vela Shell), the status of which is strongly debated (see, e.g., Sushch et al. 2011). It is not clear whether it is an enhanced part of the Gum Nebula, an interacting region with Gum, or a completely independent region in front of it.

\section{Data analysis}

As in Pagani et al. (2010), we have downloaded the Spitzer InfraRed Array Camera (IRAC, covering the range 3.6 to $8 \mu \mathrm{m}$ in four channels, Fazio et al. 2004), post-basic calibration data products of all clouds that we were able to identify in the vicinity of the Gum/Vela region. We cross-checked the IRAC images with optical digitized Schmidt plates from various surveys to identify the extent of the clouds using Aladin (Bonnarel et al. 2000). We were able to retrieve 24 sources. Eight other sources were investigated, but rejected either because of a bright star saturating the images, total absence of signal in all four IRAC bands, or wrong coordinates. These are BHR17, BHR20, 
BHR23, BHR35, BHR45, BHR48, BHR49, and BHR 50 (names from Bourke et al. 1995). Many of the sources come from the programs "Lonely Cores: Star Formation in Isolation" (PI: Bourke, T., PID 50477) and "From Molecular Cores to Planets" (PI : Evans, N., PID 139). For each source, we visually inspected the 3.6, 4.5, 5.8, and $8 \mu \mathrm{m}$ IRAC images plus the optical images. We classified the type of each cloud as A (absorption at $8 \mu \mathrm{m}$ plus absorption or nothing visible at $3.6 \mu \mathrm{m}$ ), $\mathrm{P}$ (PAH emission, i.e. emission at 3.6, 5.8 and $8 \mu \mathrm{m}$, but not at $4.5 \mu \mathrm{m}$, though there are a few cases of emission in all bands, in bright rimmed clouds - BRC -, which are a mixture of PAHs, ionized gas emission and possibly some diffraction in the optical, or excited $\mathrm{H}_{2}$ bands, especially in the case of outflows and $\mathrm{HH}$ objects, Reach et al. 2006) and C (coreshine, i.e. emission at $3.6 \mu \mathrm{m}$, possibly at $4.5 \mu \mathrm{m}$ and absorption at $8 \mu \mathrm{m}$, with nothing or absorption at $5.8 \mu \mathrm{m}$, Steinacker et al. 2010). The results are presented in Table 1, a finding chart and the images of the objects are presented in Appendix B with zoomed images on the coreshine region for the six (possibly) positive cases we report in Appendix C. Cuts and extended emission modeling (using a background evaluation function to remove stars efficiently) were performed for the most difficult cases (see Figs. C.6 and C.7). For example, BHR21 (=CG4) is a mixture of bright rimmed emission visible at all wavelengths with coreshine appearing in the center of the cometary globule (seen as a dark patch in the visible image, Fig. B.4). BHR14 and BHR 40 are two other difficult cases (Figs. B.3, B.17), with a possible mixture of coreshine and bright rimmed emission. We kept BHR14 as a possible case of coreshine emission, but rejected BHR40.

\section{Discussion}

One quarter (six) of the objects are cometary globules (Reipurth 1983; Zealey et al. 1983; Sridharan 1992; Woermann et al. 2001). Half (12) of the objects have associated IRAS sources, of which eight are known Class 0, 0/I or I objects (for a definition of Class 0 to Class III objects, see Andre et al. 2000, Sect. III.A) and two of them have associated Herbig-Haro (HH) objects. One object is a Class II (or classical T Tauri star, cTT) and two IRAS sources are chance encounter or alignement (Reipurth 1983). Four objects have no known distance and are not discussed any further. A majority of them is associated to Vela and therefore given the same distance ( $\sim 00$ pc, Reipurth 1983; Zealey et al. 1983 ), though this is probably very approximate (two of these objects, BHR34 and BHR44, have been recently brought down to 200 pc by Racca et al. 2009). It is remarkable though that most of the objects belonging to the Gum region are at a distance from the Gum center in the range $70-130$ pc, which is consistent with the determination by Yamaguchi et al. (1999, their Fig. 10) based on an independent analysis of the radial velocities of the clouds. If we consider that the outer shell radius of the Gum/Vela nebula is typically at $130 \mathrm{pc}$ from its center, we find that out of 20 clouds with known distances, 14 are inside the nebula, four are uncertain (because BHR21 is a cometary globule, it should be part of the Gum Nebula complex and is not counted as uncertain despite a possible short distance of $300 \mathrm{pc}$. Rebull et al. 2011 slightly favored the 500 pc value). Coreshine is detected in three objects and possibly three more (see Table 1, and Appendix $\mathrm{C}$ for zoomed pictures of these objects) while eight objects present $\mathrm{PAH}$ emission (twice in combination with BRC) and one object is a pure BRC case (a ninth object with PAH emission has an unknown distance). Three objects have in common $\mathrm{PAH} / \mathrm{BRC}$ emission and coreshine emission (this is only tentative for two of them). Two (BHR7 and BHR21, and possibly two more, BHR14 and DC257.3), which show coreshine effect are inside the SNR, and the last two (BHR56 and probably BHR34) are outside. This is markedly different from the results of our previous survey (Pagani et al. 2010), for which we had 7-8 times more coreshine detections than $\mathrm{PAH} /$ bright-rimmed detections and 50-60\% more coreshine detections among the investigated objects. Here, the amount of $\mathrm{PAH} / \mathrm{BRC}$ cases (eight, not counting the one with unknown distance) is comparable to, or larger than, the coreshine detections $(3+3$ potential). It is remarkable that despite the few objects found outside the SNR, two show coreshine, reminescent of the $50 \%$ detection elsewhere in the galaxy, while inside the SNR, the detection is markedly lower, between $12-25 \%$, though difficult to quantify given the uncertainty on the distances and actual detections. Therefore, the coreshine effect is rare inside the Gum Nebula. This is consistent with the destruction of big grains and the overproduction of PAHs that is expected and witnessed in slowly expanding supernovae (Andersen et al. 2011). This confirms the status of the Gum Nebula as an SNR.

Though the exact center and exact extent of the SNR are difficult to establish, it seems relatively secure to state that the entire internal part of the SNR is void of molecular clouds. Except for DC257.3-2.5, which is estimated to be at $30 \mathrm{pc}$ from the center, all other clouds lie at distances above $60 \mathrm{pc}$. The clouds inside this radius have therefore been either completely evaporated or were pushed away. If all clouds had been pushed away, one would expect to find among them comparatively many clouds with traces of coreshine, which is not the case. Most clouds inside the $60 \mathrm{pc}$ radius must therefore have dispersed upon the passage of the blast wave, though one would expect the blast wave to have lost most of its energy at such a distance from the center. The clouds beyond $60 \mathrm{pc}$ have resisted evaporation but many have partially collapsed to form stars. Indeed, Bhatt (1993) has shown that there is an enhanced star formation among the cometary globules belonging to the Gum Nebula region. It is also remarkable that several of the observed clouds contain protostars or IRAS objects without associated coreshine. This is again markedly different from the results of the galactic survey (Pagani et al. 2010), which gave $75 \%$ of coreshine detections out of 16 objects with buried protostar (very low luminosity objects or VeLLO, Class 0, Class I). If we understand these clouds as clouds that have formed stars due to the passing of the shock wave and that the shock wave has also shattered the big grains, it is conceivable that the time has been long enough to build the stars, but not long enough to regrow big grains. Those few we see might be survivors left over from the blast wave passage or the first ones that have reached sizes large enough for efficient MIR scattering after the initial destruction. Most of the infrared sources being Class 0 or Class I objects, their age is less than a few hundred thousand years (Ward-Thompson et al. 2007), i.e. much less than the age of the SNR. Because these clouds are far from the center of the SNR, it is quite probable that they have been reached by the blast wave relatively recently. This combination of Class I objects and absence of coreshine gives us a lower limit to the necessary time to build micron-size grains consistent with the numerical modeling that requires several hundred thousand years to gain a factor 10 in size (this depends somewhat on the conditions, though. See Ormel et al. 2009). Therefore, in the other star-forming regions of the Galaxy, either the clouds have lived long enough to build up big grains before the protostars form or other cloud properties/initial conditions dominate the apparition of the effect. In particular, this probably indicates that most of these clouds never suffered from 
Table 1. List of dark clouds and cometary globules in the vicinity of the Gum Nebula.

\begin{tabular}{|c|c|c|c|c|c|c|}
\hline Name & Right ascension & Declination & Sun distance $^{a}$ & Gum distance ${ }^{b}$ & Type $^{c}$ & Embedded object $^{d}$ \\
\hline & hh:mm:ss (J2000) & dd:mm:ss (J2000) & Parsec & Parsec & & \\
\hline $\mathrm{BHR} 22=\mathrm{CG} 13$ & $07: 14: 11.000$ & $-48: 31: 00.00$ & $400 *$ & 110 & $P$ & $\mathrm{I}^{e}$ \\
\hline BHR14 = CG34 & $07: 29: 32.7$ & $-41: 10: 30$ & $400 *$ & 90 & $\mathrm{P}(+\mathrm{C} ?)$ & $\mathrm{N}$ \\
\hline $\mathrm{BHR} 21=\mathrm{CG} 4$ & 07:34:09.0 & $-46: 54: 18.00$ & $300 / 500$ & $160 / 90$ & $\mathrm{C}+\mathrm{P}$ & $\mathrm{C} 1 ?$ \\
\hline BHR16 & $08: 05: 26.0$ & $-39: 08: 54.0$ & 250 & 200 & A & $\mathrm{N}$ \\
\hline BHR9-12 = CG30/31 & 08:09:04.000 & $-36: 00: 51.00$ & $400 *$ & 70 & $\mathrm{P}$ & $\mathrm{C} 0 / 1+\mathrm{HH} 120$ \\
\hline $\mathrm{BHR} 7=\mathrm{CG} 32$ & $08: 14: 21.892$ & $-34: 31: 08.41$ & $400 *$ & 80 & $\mathrm{C}$ & $\mathrm{C} 0 / 1$ \\
\hline DC257.3-2.5 & $08: 17: 09.31$ & $-39: 52: 25.00$ & 440 & 30 & $\mathrm{~A}(+\mathrm{C} ?)$ & $\mathrm{I}^{\mathrm{f}}$ \\
\hline BHR31 & $08: 18: 47.272$ & $-49: 42: 34.40$ & $400 *$ & 70 & A & I \\
\hline DC266.0-7.5 & $08: 21: 22.941$ & $-49: 50: 27.16$ & $400 *$ & 70 & A & $\mathrm{N}$ \\
\hline BHR36 & $08: 25: 40.933$ & $-51: 00: 50.66$ & $400 / 450$ & $90 / 70$ & A & $\mathrm{C} 1+\mathrm{HH} 46 / 47$ \\
\hline BHR42 & $08: 26: 21.265$ & $-51: 39: 23.42$ & ? & - & $\mathrm{P}$ & - \\
\hline BHR37 & 08:26:31.999 & $-51: 02: 18.25$ & $400 *$ & 90 & $\mathrm{~A}+\mathrm{P}$ & $\mathrm{N}$ \\
\hline BHR41 & $08: 27: 28.046$ & $-51: 09: 45.82$ & $400 *$ & 90 & A & $\mathrm{C} 1$ \\
\hline BHR13 = CG22 & $08: 28: 44.0$ & $-33: 45: 12$ & $400 *$ & 90 & A & $\mathrm{C} 2$ \\
\hline BHR34 & $08: 31: 48.846$ & $-50: 35: 40.89$ & $200 / 400 *$ & $250 / 80$ & $\mathrm{P}(+\mathrm{C} ?)$ & $\mathrm{CO}$ \\
\hline BHR40 & $08: 31: 58.164$ & $-50: 34: 37.85$ & ? & - & $\mathrm{P}$ & - \\
\hline BHR38/39 & $08: 34: 22.589$ & $-50: 22: 23.22$ & 450 & 70 & $\mathrm{P}$ & $\mathrm{N}$ \\
\hline BHR56 & $08: 44: 02.664$ & $-59: 53: 41.03$ & $400 *$ & 130 & $\mathrm{C}$ & $\mathrm{CO}$ \\
\hline BHR30 & $08: 53: 43.0$ & $-44: 48: 00.0$ & $?$ & - & A & $\mathrm{N}$ \\
\hline BHR43 & $09: 22: 23.681$ & $-45: 54: 26.86$ & $400 *$ & 100 & A & $\mathrm{N}$ \\
\hline BHR44 & $09: 26: 15.978$ & $-45: 11: 01.84$ & $200 / 400 *$ & $260 / 100$ & A & $\mathrm{N}$ \\
\hline BHR53 & $09: 28: 47.896$ & $-51: 35: 38.60$ & $200 / 500$ & $260 / 130$ & A & $\mathrm{N}$ \\
\hline BHR47 & $09: 31: 02.8$ & $-48: 38: 22.00$ & $?$ & - & A & - \\
\hline BHR55 & 09:46:51.00 & $-51: 06.4$ & 300 & 190 & A & $\mathrm{C} 0$ \\
\hline
\end{tabular}

Notes. ${ }^{(a)}$ A * indicates that the object is associated to Vela (Reipurth 1983; Zealey et al. 1983). Other distances are obtained from the analysis of the star reddening (Bourke et al. 1995; Lee \& Myers 1999; Löhr et al. 2007). Three objects have no known distance. ${ }^{(b)}$ The distance to the Gum Nebula center is computed assuming the coordinates to be $\alpha_{\mathrm{J} 2000}=08^{\mathrm{h}} 22^{\mathrm{m}} 53.6^{\mathrm{s}}, \delta_{\mathrm{J} 2000}=-42^{\circ} 25^{\prime} 20^{\prime \prime}$ and a distance to the Sun of $450 \mathrm{pc}$. (c) P: PAH emission (or BRCs, see text. Emission in both 3.6 and $8 \mu \mathrm{m}$ bands), C: coreshine (emission at 3.6 and absorption at $8 \mu \mathrm{m}$ ), A: absorption (at $8 \mu \mathrm{m}$, nothing or absorption at $3.6 \mu \mathrm{m}) .{ }^{(d)} \mathrm{N}$ : no associated object, I: IRAS object, C0: Class 0 object, C1: Class I object, C2: Class II (classic T Tauri) object. See Sahu \& Sahu (1993); Henning \& Launhardt (1998); Santos et al. (1998); Racca et al. (2009); Lahuis et al. (2010); Launhardt et al. (2010); Rebull et al. (2011). ${ }^{(e)}$ A chance encounter with a field star (Reipurth 1983). ${ }^{(f)}$ Probably a chance alignment with a field star.

a shock wave or a radiation-driven implosion. Combined with the age upper limit of a few million years that we can derive from the absence of $\mathrm{DCO}^{+}$in dark clouds outside the dense and CO-depleted cores (Pagani et al. 2011), we start to be able to bracket the age of individual clouds.

\section{Conclusions}

We confirm that coreshine is almost absent from the Gum Nebula clouds, it is detected with certainty in only two objects out of 14 (possibly 18) inside the Gum region, two others being questionable while the number of objects with PAH or BRC emission is at least comparable or even larger, in marked contrast with the general survey of the Galaxy. The absence of clouds inside the inner half of the Gum region, the many young stellar objects in the outer half combined with an almost total absence of coreshine, again in marked contrast with the general survey of the Galaxy, confirm the SNR nature of the Gum Nebula. The absence of coreshine combined with the presence of Class I objects is consistent with the passage of a blast wave in the recent past to both destroy the big grains and force the clouds to collapse to form stars. Given the very old age of the SNR itself, this can be explained only because the clouds are far from the SNR center and the blast wave only reached them recently.

Acknowledgements. This work is based on observations made with the Spitzer Space Telescope, which is operated by the Jet Propulsion Laboratory, California Institute of Technology, under NASA contract 1407. This work has made heavy use of Aladin and Simbad (Bonnarel et al. 2000). We wish to thank the referee for her/his careful reading of the manuscript and suggested improvements.

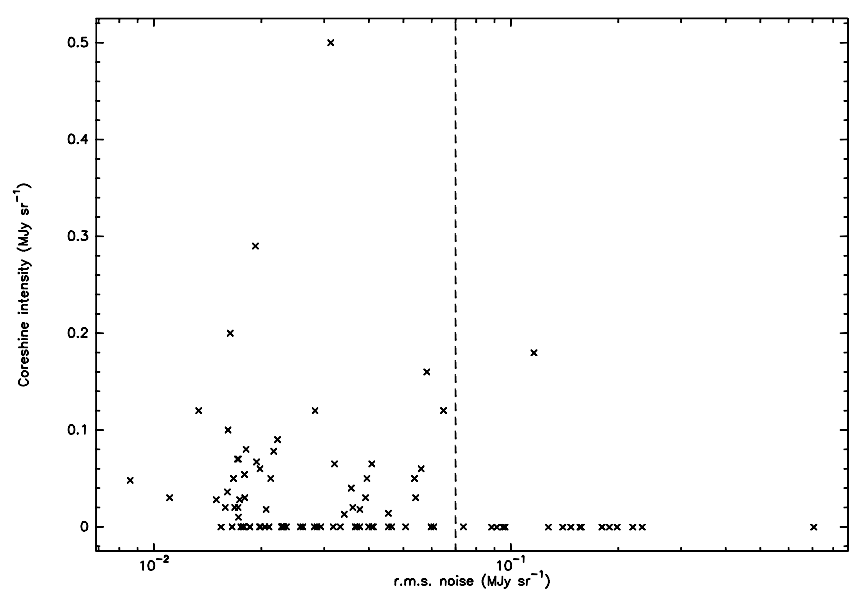

Fig. A.1. Coreshine intensity as a function of IRAC $3.6 \mu \mathrm{m}$ noise level. The vertical dashed line situated at $0.07 \mathrm{MJy} \mathrm{sr}^{-1}$ sets the limit above which absence of coreshine might be due to high noise or high background level only and not intrinsic absence of big grains.

\section{Appendix A: Detectability of coreshine}

In Pagani et al. (2010), we did not discuss the possible limitation of coreshine detection due to high noise levels. After finding more than $50 \%$ of positive cases, the loss of a few cases due to high noise would not have changed the result dramatically and of course, not all negative cases had high noise level. Conversely, it is interesting to know what the noise level limit is 
above which no coreshine was detected to check that the present study is not limited only by noise. In Fig. A.1 we show the intensity of the coreshine effect as a function of noise for $92 \mathrm{ob}-$ jects out of the 110 objects considered in the Pagani et al. (2010) study in the IRAC $3.6 \mu \mathrm{m}$ band. We have removed the objects whose data were useless and the objects in the Gum vicinity to set up an independent sample. We find that apart from one case, B59, with both strong coreshine $\left(0.15 \mathrm{MJy} \mathrm{sr}^{-1}\right)$ and noise $\left(0.12 \mathrm{MJy} \mathrm{sr}^{-1}\right)$, there is no coreshine detection for objects with noise above $0.07 \mathrm{MJy} \mathrm{sr}^{-1}$. There are 17 objects (including B59) out of 92 above the limit. Most of these objects show absorption at $8 \mu \mathrm{m}$, none shows emission (either coresine or PAHs except B59) and three show nothing. For these objects we cannot say that coreshine is totally excluded but we cannot see it if present. These objects also present high background, which is the main cause for their high noise, which also prevents the possible detection of coreshine. There are also two objects with low noise but high background (CB44 and $\rho$ Oph D), which do not show coreshine, possibly for the same reasons. All observations in the present study have a noise below the limit of $0.07 \mathrm{MJy} \mathrm{sr}^{-1}$ and are therefore considered as reliable to determine the presence of coreshine in them. We could have set up the limit above the B59 case $\left(0.12 \mathrm{MJy} \mathrm{sr}^{-1}\right)$, but this would have only weakened the limit constraint. Our present more stringent limit gives us confidence that the Gum/Vela observations we retrieved from the archive are all eligible to evaluate the presence of coreshine in this region.

\section{References}

Andersen, M., Rho, J., Reach, W. T., Hewitt, J. W., \& Bernard, J. P. 2011, ApJ, 742, 7

Andre, P., Ward-Thompson, D., \& Barsony, M. 2000, Protostars and Planets IV, 59

Arendt, R. G., Dwek, E., Blair, W. P., et al. 2010, ApJ, 725, 585

Beuermann, K. P. 1973, Ap\&SS, 20, 27

Bhatt, H. C. 1993, MNRAS, 262, 812

Bonnarel, F., Fernique, P., Bienaymé, O., et al. 2000, A\&AS, 143, 33

Borkowski, K. J., \& Dwek, E. 1995, ApJ, 454, 254

Bourke, T. L., Hyland, A. R., \& Robinson, G. 1995, MNRAS, 276, 1052

Brandt, J. C., Stecher, T. P., Crawford, D. L., \& Maran, S. P. 1971, ApJ, 163, L99

Chanot, A., \& Sivan, J. P. 1983, A\&A, 121, 19

Choudhury, R., \& Bhatt, H. C. 2009, MNRAS, 393, 959
Fazio, G. G., Hora, J. L., Allen, L. E., et al. 2004, ApJS, 154, 10

Franco, G. A. P. 1990, A\&A, 227, 499

Guillet, V., Pineau Des Forêts, G., \& Jones, A. P. 2007, A\&A, 476, 263

Guillet, V., Jones, A. P., \& Pineau Des Forêts, G. 2009, A\&A, 497, 145

Guillet, V., Pineau des Forêts, G., \& Jones, A. P. 2011, A\&A, 527, A123

Gum, C. S. 1952, The Observatory, 72, 151

Henning, T., \& Launhardt, R. 1998, A\&A, 338, 223

Hippelein, H. H., \& Weinberger, R. 1975, A\&A, 43, 405

Jones, A. P., Tielens, A. G. G. M., Hollenbach, D. J., \& McKee, C. F. 1994, ApJ, 433, 797

Jones, A. P., Tielens, A. G. G. M., \& Hollenbach, D. J. 1996, ApJ, 469, 740

Lahuis, F., van Dishoeck, E. F., Jørgensen, J. K., Blake, G. A., \& Evans, N. J. 2010, A\&A, 519, A3

Launhardt, R., Nutter, D., Ward-Thompson, D., et al. 2010, ApJS, 188, 139

Leahy, D. A., Nousek, J., \& Garmire, G. 1992, ApJ, 385, 561

Lee, C. W., \& Myers, P. C. 1999, ApJS, 123, 233

Löhr, A., Bourke, T. L., Lane, A. P., et al. 2007, ApJS, 171, 478

Micelotta, E. R., Jones, A. P., \& Tielens, A. G. G. M. 2010, A\&A, 510, A36

Ormel, C. W., Paszun, D., Dominik, C., \& Tielens, A. G. G. M. 2009, A\&A, 502, 845

Pagani, L., Steinacker, J., Bacmann, A., Stutz, A., \& Henning, T. 2010, Science, 329, 1622

Pagani, L., Roueff, E., \& Lesaffre, P. 2011, ApJ, 739, L35

Racca, G. A., Vilas-Boas, J. W. S., \& de la Reza, R. 2009, ApJ, 703, 1444

Rajagopal, J., \& Srinivasan, G. 1998, J. Astrophys. Astron., 19, 79

Reach, W. T., Rho, J., Tappe, A., et al. 2006, AJ, 131, 1479

Rebull, L. M., Johnson, C. H., Hoette, V., et al. 2011, AJ, 142, 25

Reipurth, B. 1983, A\&A, 117, 183

Reynolds, R. J. 1976a, ApJ, 203, 151

Reynolds, R. J. 1976b, ApJ, 206, 679

Reynoso, E. M., \& Dubner, G. M. 1997, A\&AS, 123, 31

Sahu, M. S. 1992, Ph.D. Thesis, Kapteyn Institute, Postbus 8009700 AV Groningen, The Netherlands

Sahu, M. S., \& Sahu, K. C. 1993, A\&A, 280, 231

Sankrit, R., Williams, B. J., Borkowski, K. J., et al. 2010, ApJ, 712, 1092

Santos, N. C., Yun, J. L., Santos, C. A., \& Marreiros, R. G. 1998, AJ, 116, 1376

Sridharan, T. K. 1992, J. Astrophys. Astron., 13, 217

Srinivasan, M., Pecker, J. C., Pottasch, S. R., \& Sahu, K. C. 1987, The Messenger, 50, 11

Steinacker, J., Pagani, L., Bacmann, A., \& Guieu, S. 2010, A\&A, 511, A9

Sushch, I., Hnatyk, B., \& Neronov, A. 2011, A\&A, 525, A154

Tenorio-Tagle, G., \& Bodenheimer, P. 1988, ARA\&A, 26, 145

Urquhart, J. S., Morgan, L. K., \& Thompson, M. A. 2009, A\&A, 497, 789

Wallerstein, G., Jenkins, E. B., \& Silk, J. 1980, ApJ, 240, 834

Ward-Thompson, D., Andre, P., Crutcher, R. M., et al. 2007, Protostars and Planets V, 33

Woermann, B., Gaylard, M. J., \& Otrupcek, R. 2001, MNRAS, 325, 1213

Yamaguchi, N., Mizuno, N., Moriguchi, Y., et al. 1999, PASJ, 51, 765

Zealey, W. J., Ninkov, Z., Rice, E., Hartley, M., \& Tritton, S. B. 1983, ApJ, 23, L119 


\section{Appendix B: Finding chart, optical and Spitzer/IRAC images of the clouds in the vicinity of Gum/Vela}

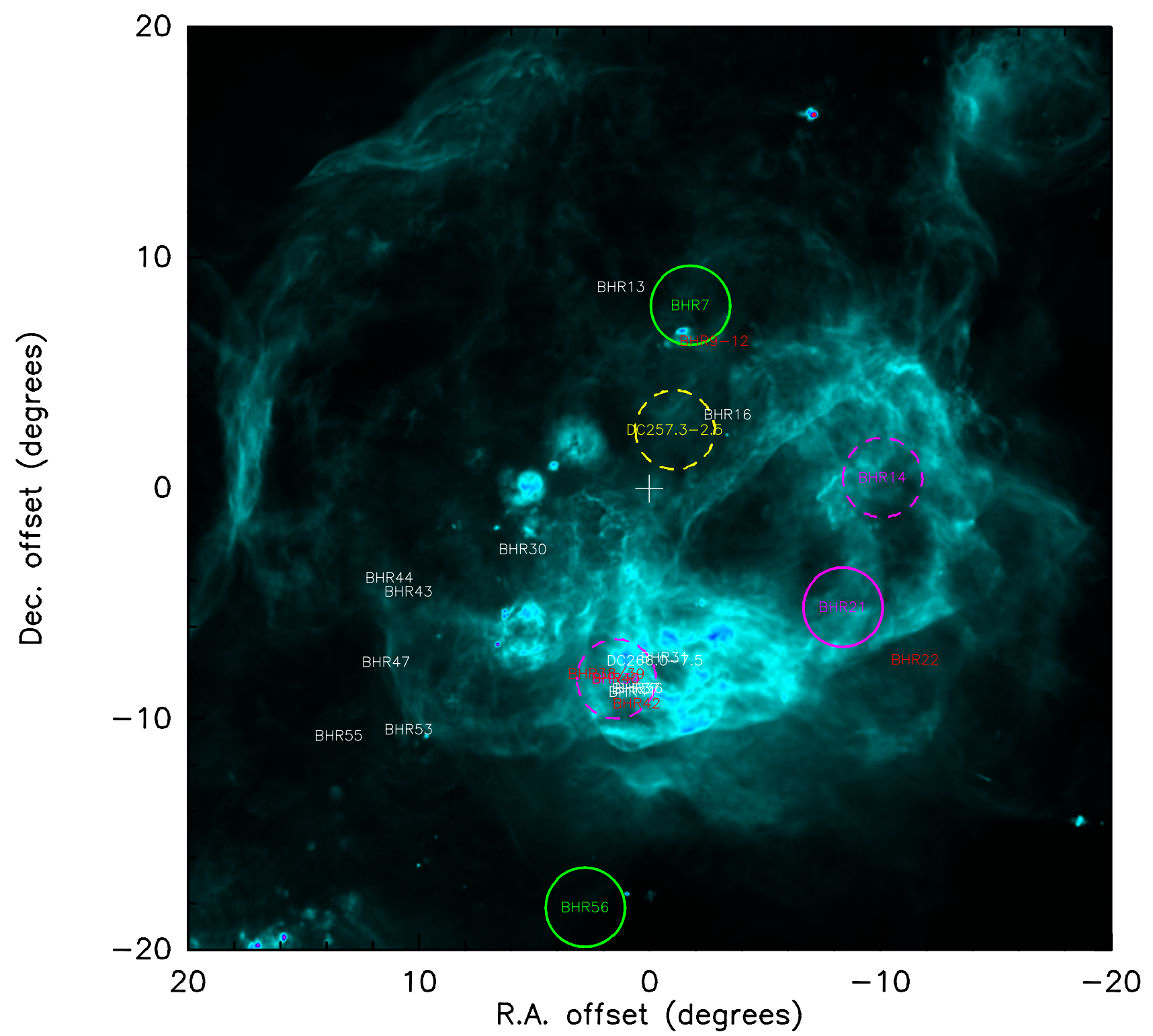

Fig. B.1. Finding chart: $\mathrm{H} \alpha$ emission of the Gum Nebula region (from NASA/Skyview) with the 24 sources indicated by their name. The color code is the following: red $=\mathrm{PAH} / \mathrm{BRC}$ emission, white $=$ absorption, green $=$ coreshine, yellow $=$ absorption + coreshine, purple $=\mathrm{PAH}+$ coreshine . Full line circles indicate clear coreshine cases, dashed line circles indicate dubious coreshine cases. The cross in the middle is the reference position $\alpha_{\mathrm{J} 2000}=08^{\mathrm{h}} 22^{\mathrm{m}} 53.6^{\mathrm{s}}, \delta_{\mathrm{J} 2000}=-42^{\circ} 25^{\prime} 20^{\prime \prime}$ for the Gum Nebula center. 
L. Pagani et al.: Absence of coreshine in the Gum/Vela region $(R N)$

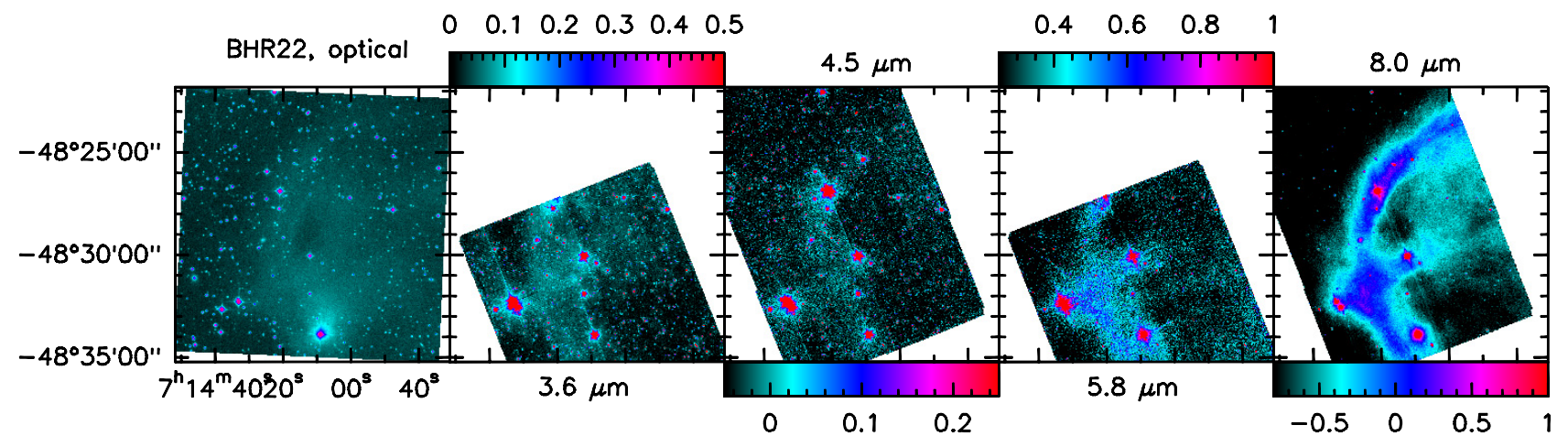

Fig. B.2. Images of BHR22 $=$ CG13. From left to right: optical, 3.6, 4.5, 5.8 and $8.0 \mu \mathrm{m}$. Optical images are digitized photographic plate portions, hence not absolutely calibrated and no color bar is given. They only help to identify the objects. Infrared image scales (MJy sr $\left.{ }^{-1}\right)$ are adjusted to maximize the contrast. Coordinates are identical in all five boxes but shown only in the first box. Type of cloud: PAH emission (or BRC, see text), presence of an IRAS object.

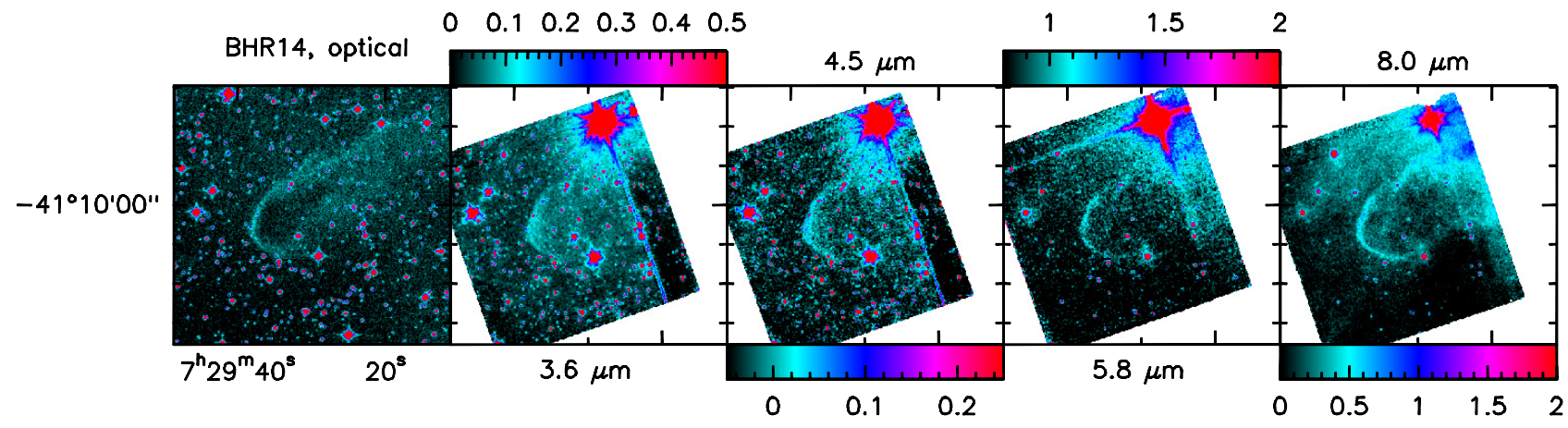

Fig. B.3. Images of BHR14 = CG34. PAH emission and possibly coreshine, no embedded object. Same as Fig. B.2.

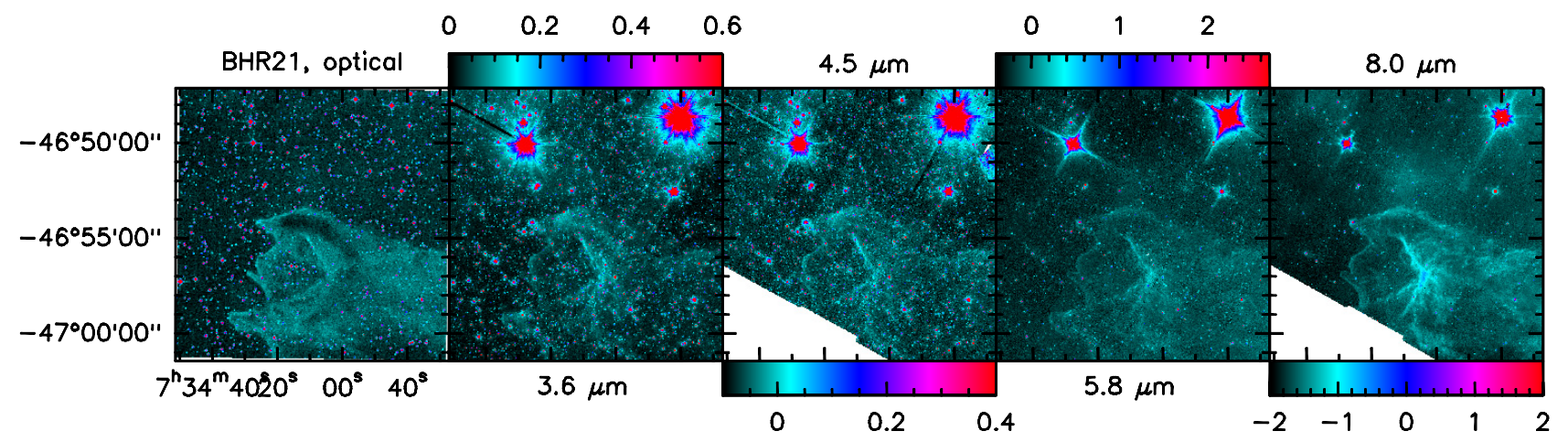

Fig. B.4. Images of BHR21= CG4. Coreshine and PAH emission, Class I object? Same as Fig. B.2.

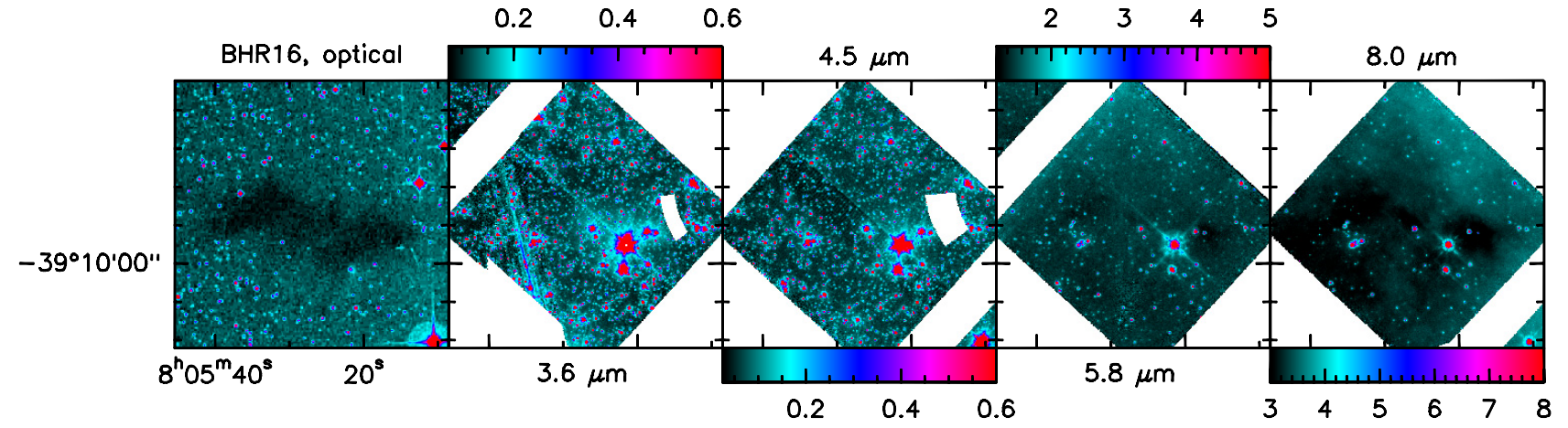

Fig. B.5. Images of BHR16. Absorption, no embedded object. Same as Fig. B.2. 


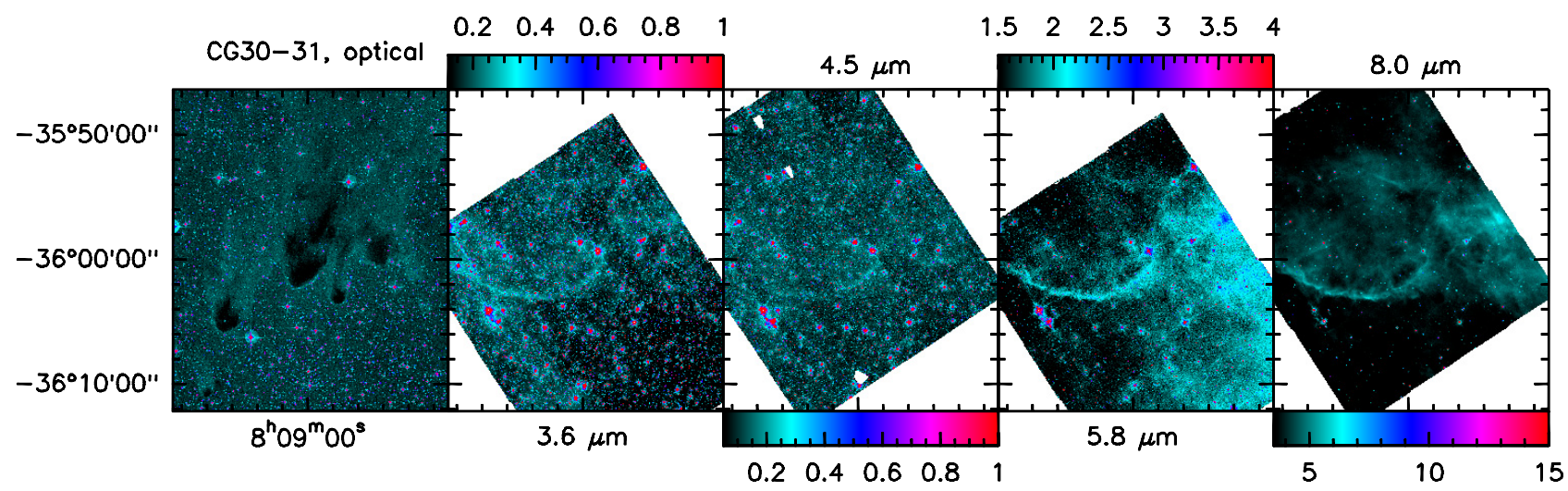

Fig. B.6. Images of CG30/31. PAH emission, Class 0/I object + HH120. Same as Fig. B.2.

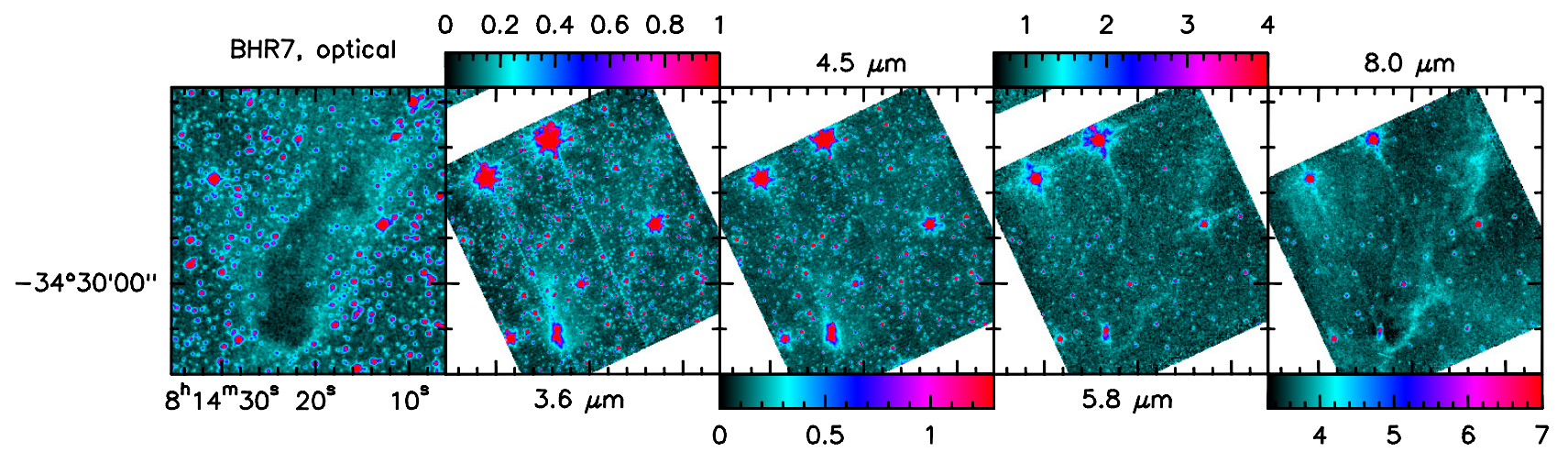

Fig. B.7. Images of BHR7. Coreshine, Class 0/I object. Same as Fig. B.2.

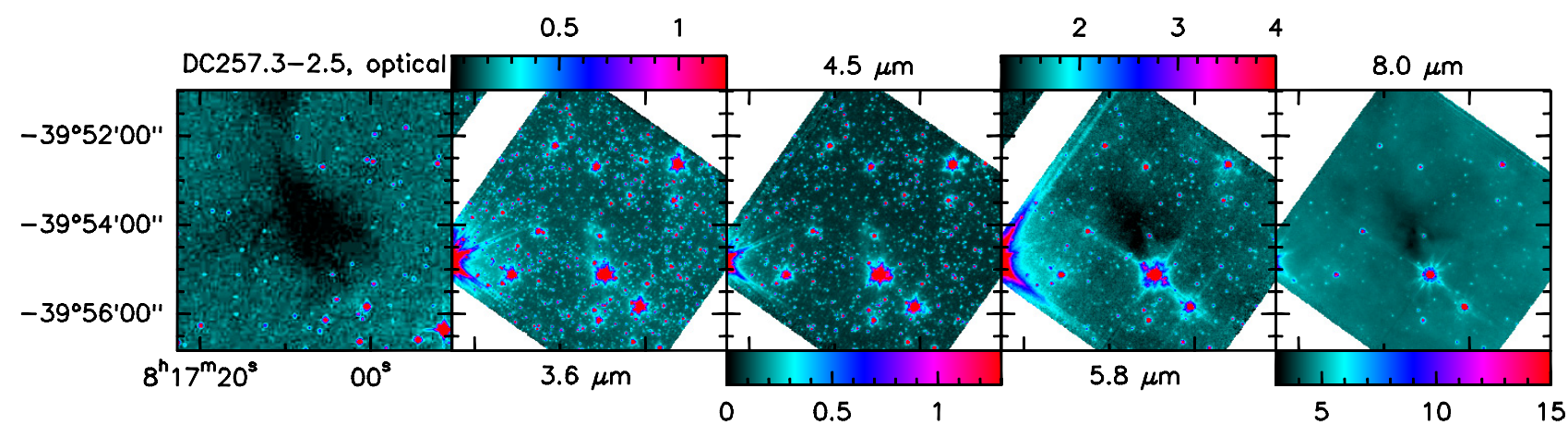

Fig. B.8. Images of DC257.3-2.5. Absorption and possibly coreshine, presence of an IRAS object. Same as Fig. B.2.

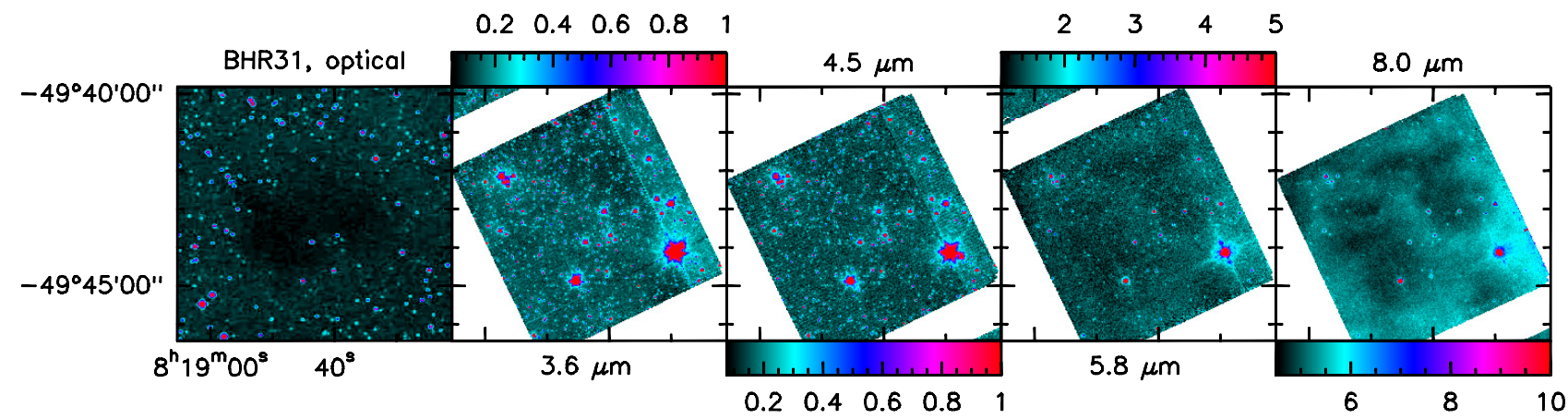

Fig. B.9. Images of BHR31. Absorption, presence of an IRAS object. Same as Fig. B.2. 
L. Pagani et al.: Absence of coreshine in the Gum/Vela region $(R N)$

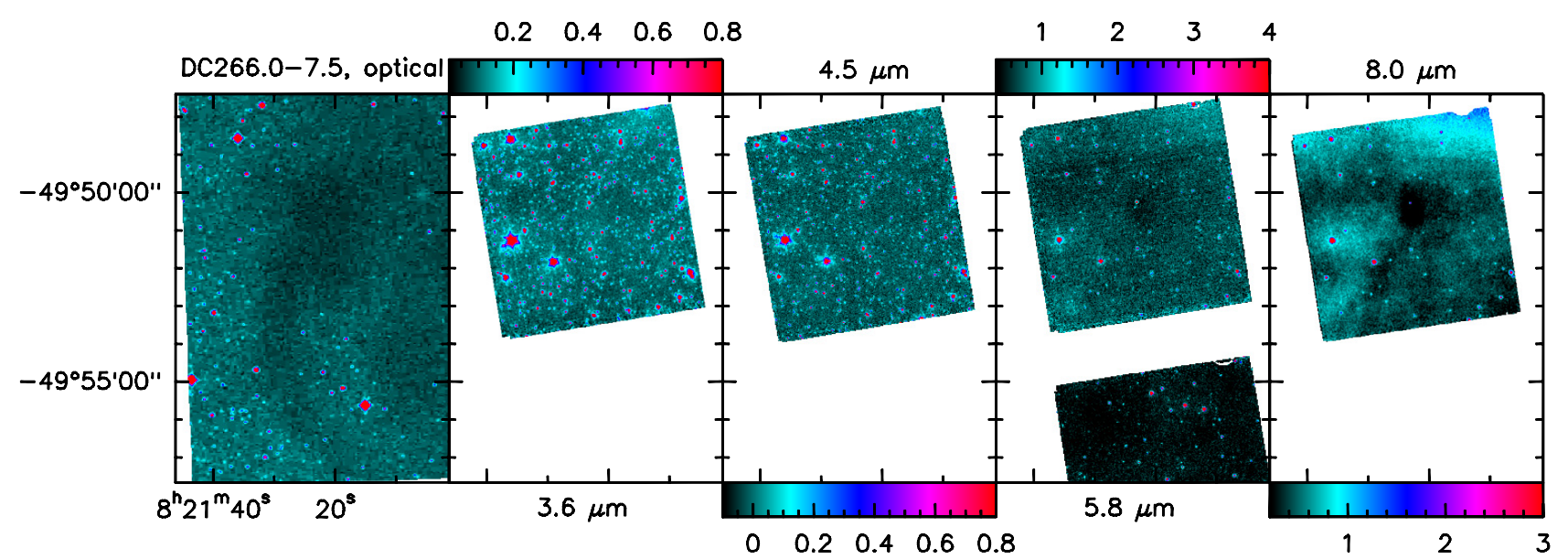

Fig. B.10. Images of DC266.0-7.5. Absorption, no embedded object. Same as Fig. B.2.

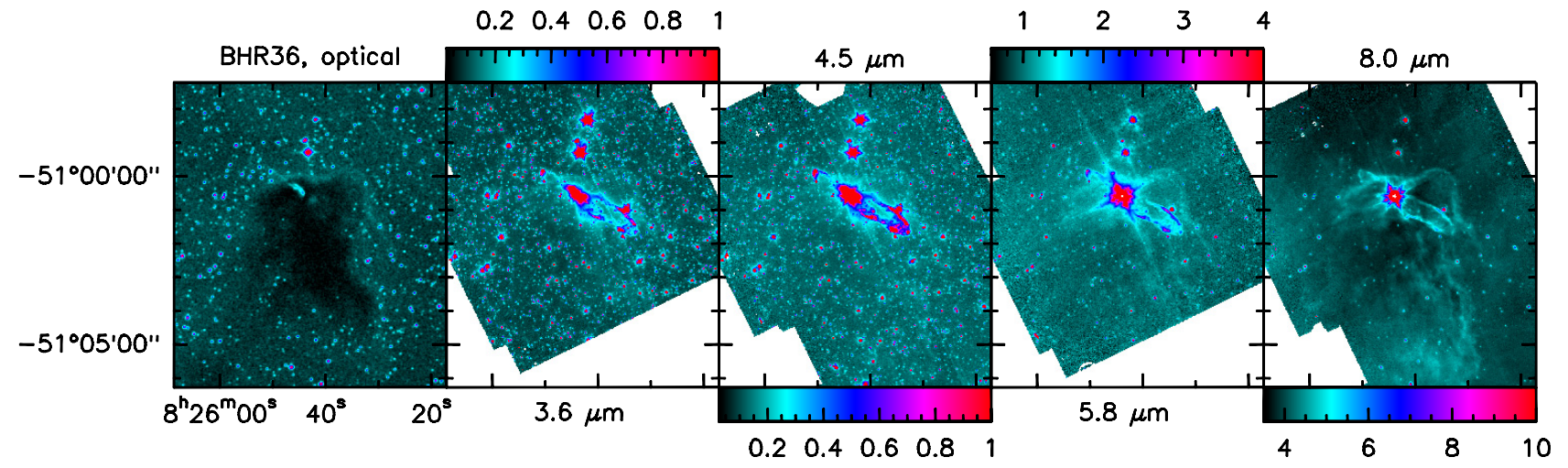

Fig. B.11. Images of BHR36. Absorption, Class I object + HH46/47. Same as Fig. B.2.

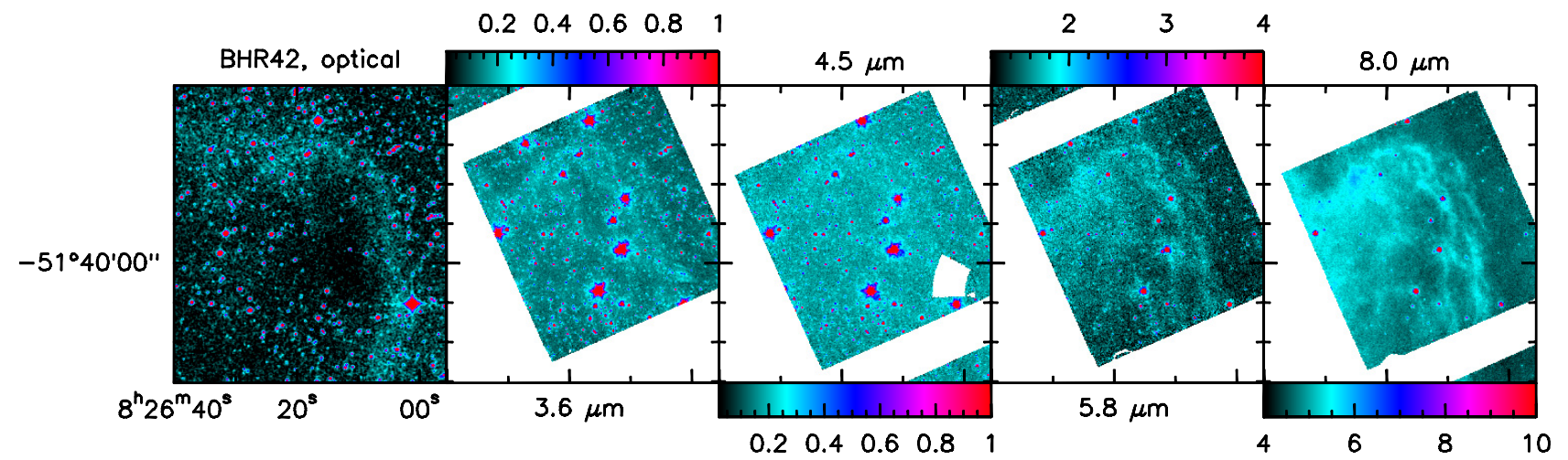

Fig. B.12. Images of BHR42. PAH emission. Same as Fig. B.2. 


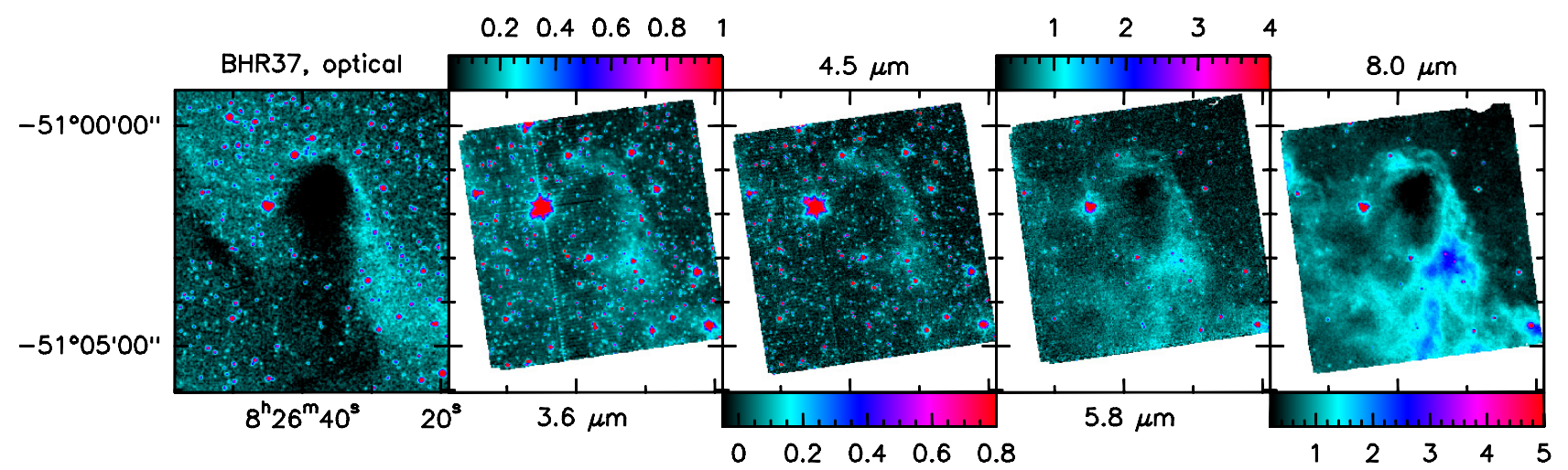

Fig. B.13. Images of BHR37. Absorption and PAH emission, no embedded object. Same as Fig. B.2.

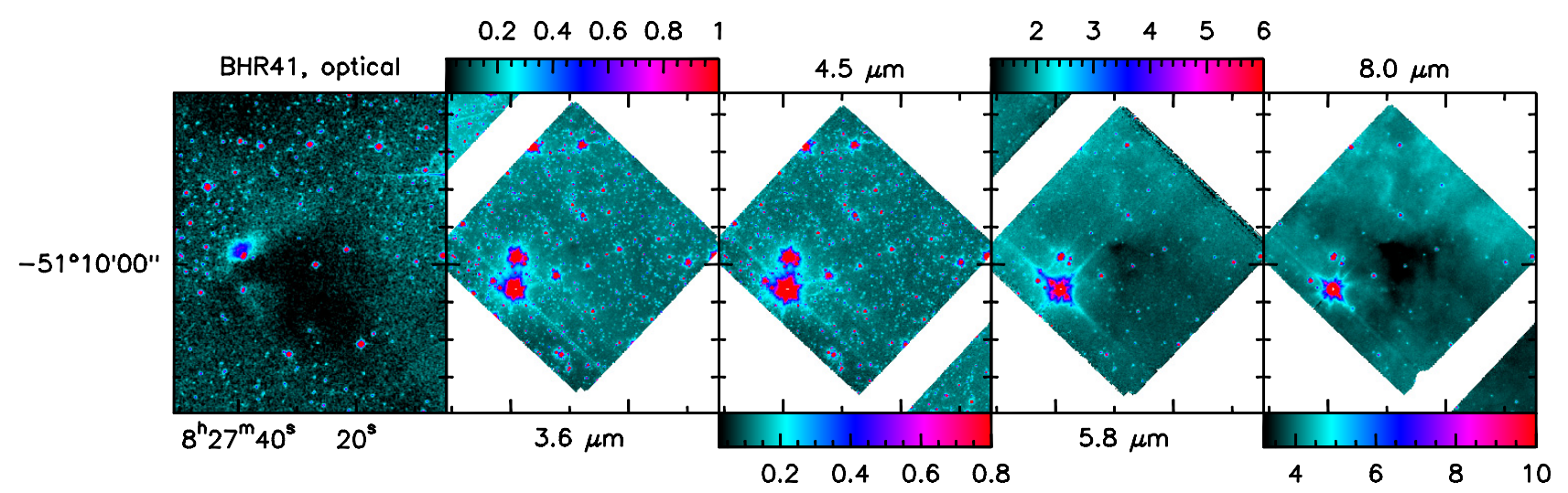

Fig. B.14. Images of BHR41. Absorption, Class I object. Same as Fig. B.2.

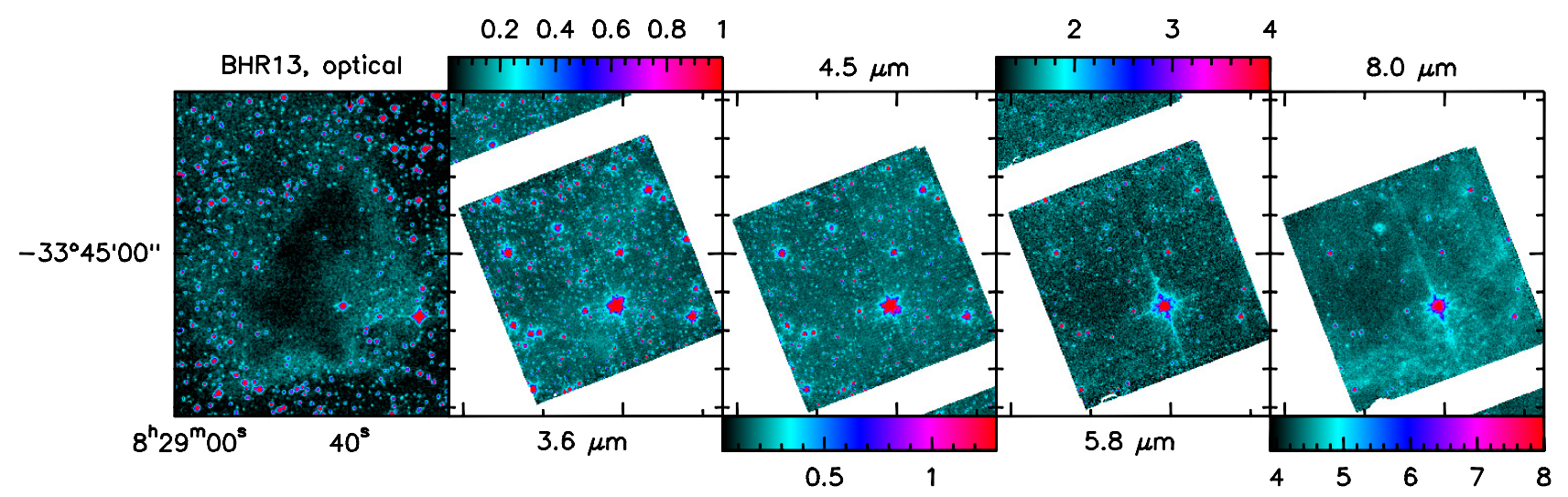

Fig. B.15. Images of BHR13 = CG22. Absorption, Class II object. Same as Fig. B.2. 
L. Pagani et al.: Absence of coreshine in the Gum/Vela region $(R N)$

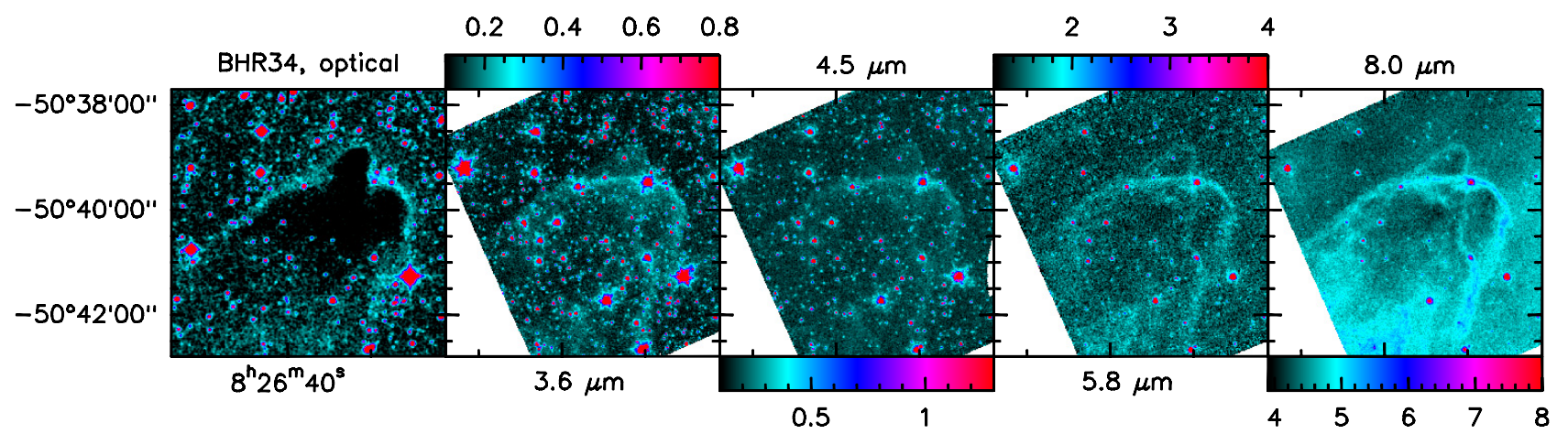

Fig. B.16. Images of BHR34. PAH emission and possibly coreshine, Class I object? Same as Fig. B.2.

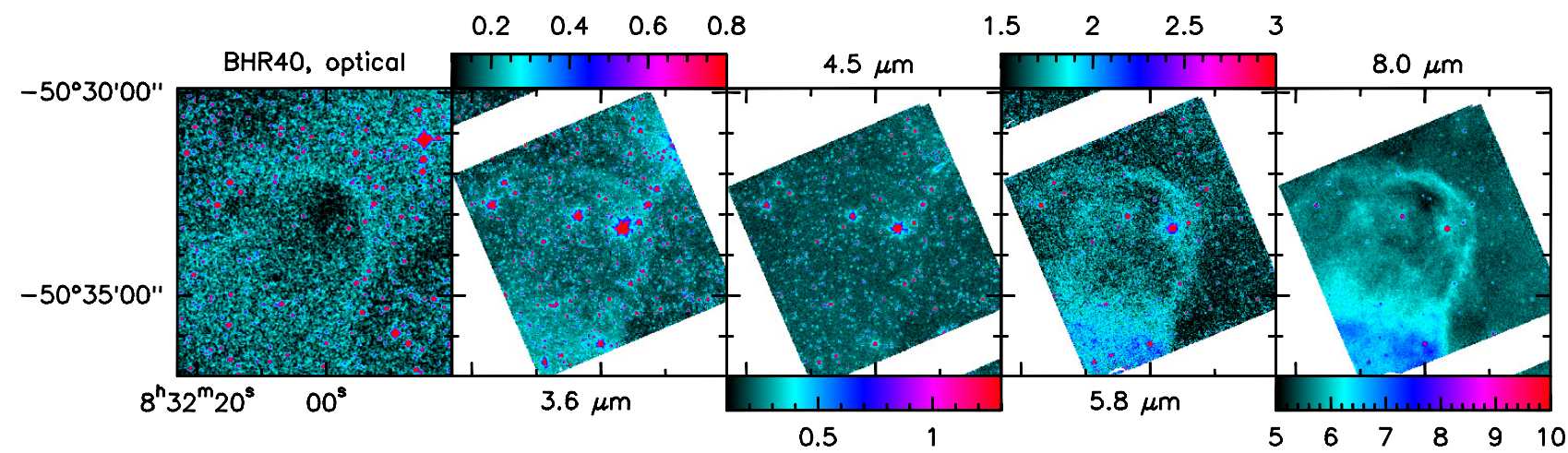

Fig. B.17. Images of BHR40. PAH emission. Same as Fig. B.2.

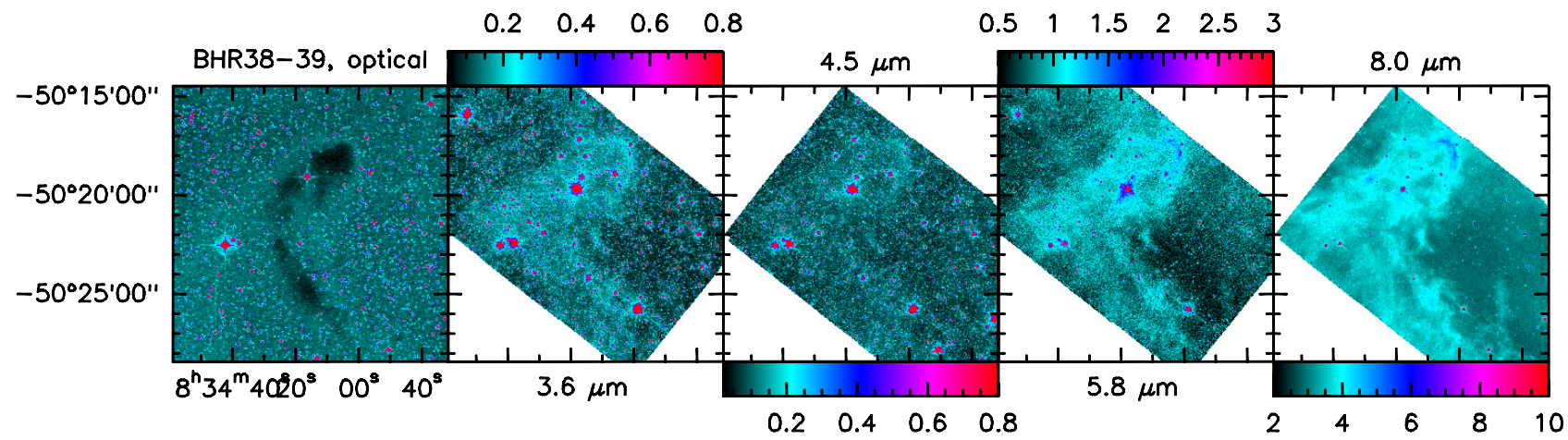

Fig. B.18. Images of BHR38/39. PAH emission, no embedded object. Same as Fig. B.2.

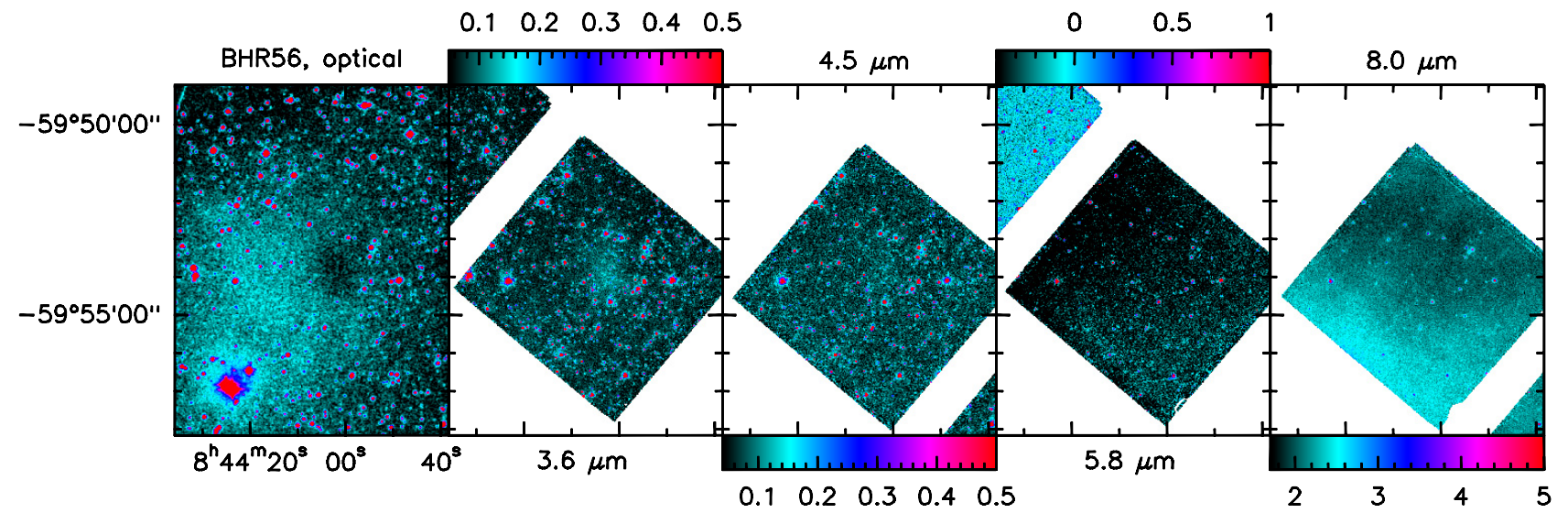

Fig. B.19. Images of BHR56. Coreshine, Class 0 object. Same as Fig. B.2. 


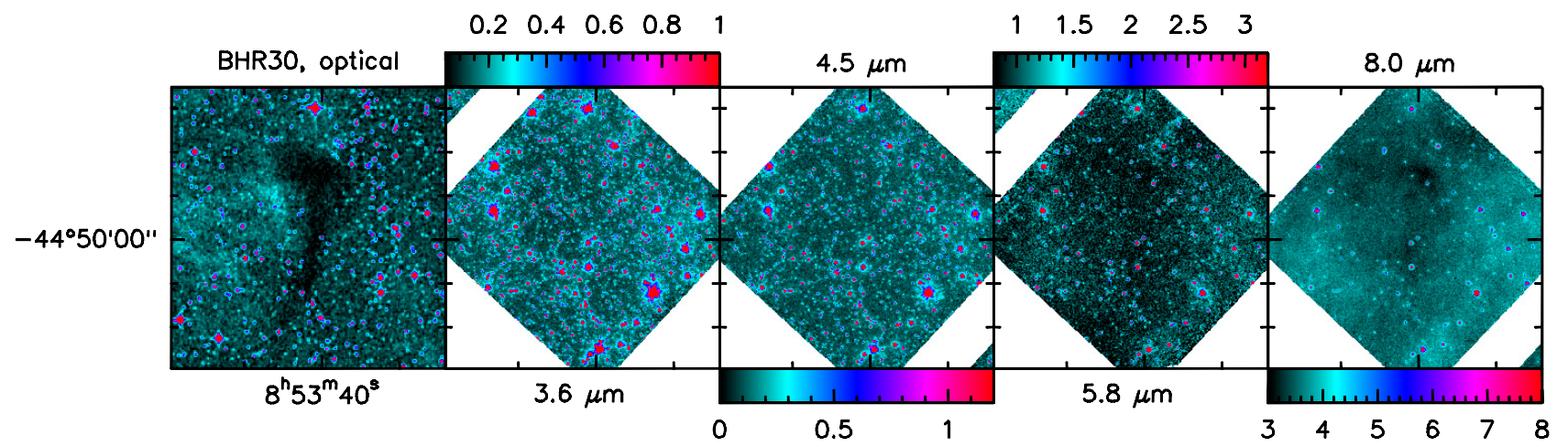

Fig. B.20. Images of BHR30. Absorption, no embedded object. Same as Fig. B.2.

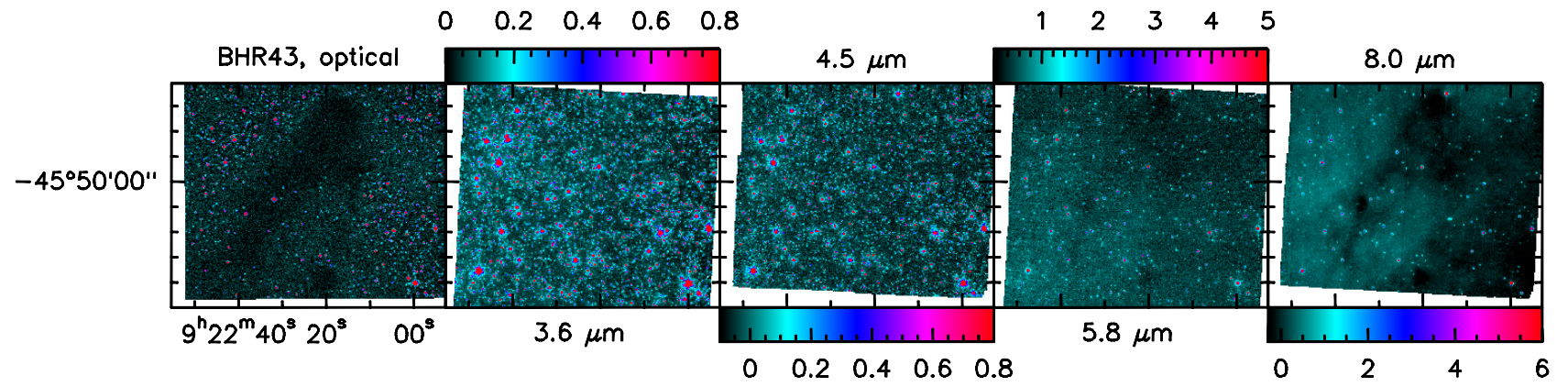

Fig. B.21. Images of BHR43. Absorption, no embedded object. Same as Fig. B.2.

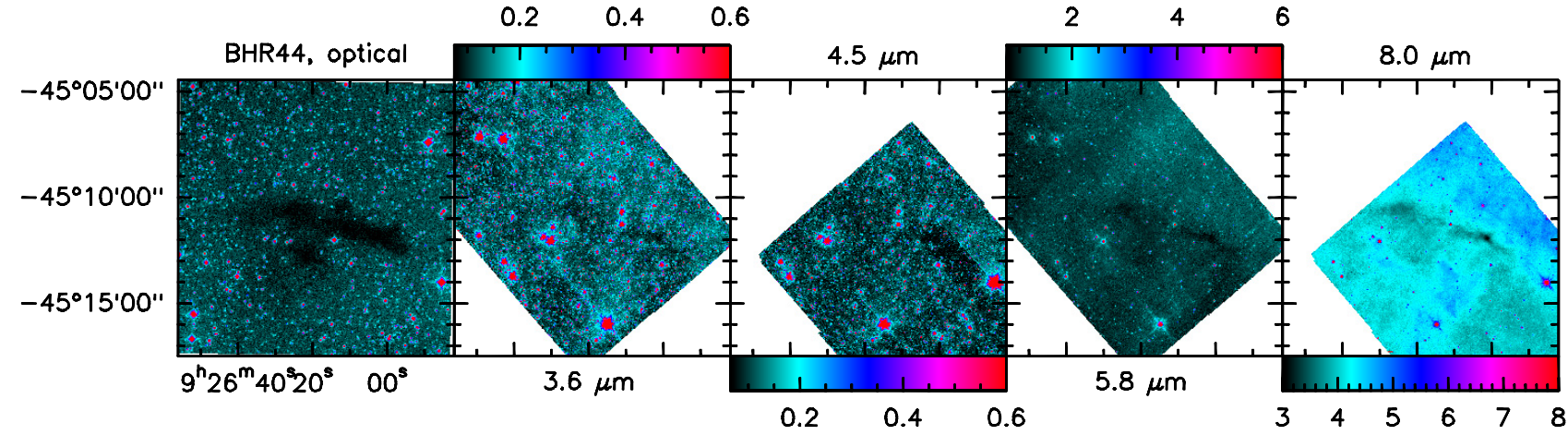

Fig. B.22. Images of BHR44. Absorption, no embedded object. Same as Fig. B.2.

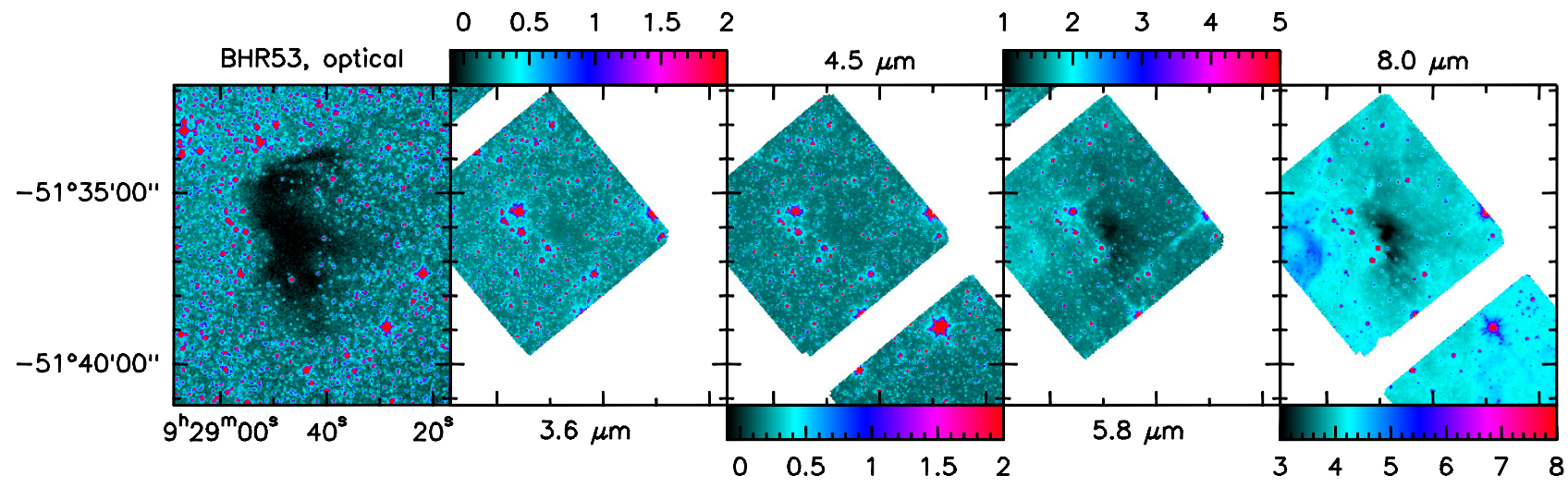

Fig. B.23. Images of BHR53. Absorption, no embedded object. Same as Fig. B.2. 
L. Pagani et al.: Absence of coreshine in the Gum/Vela region $(R N)$

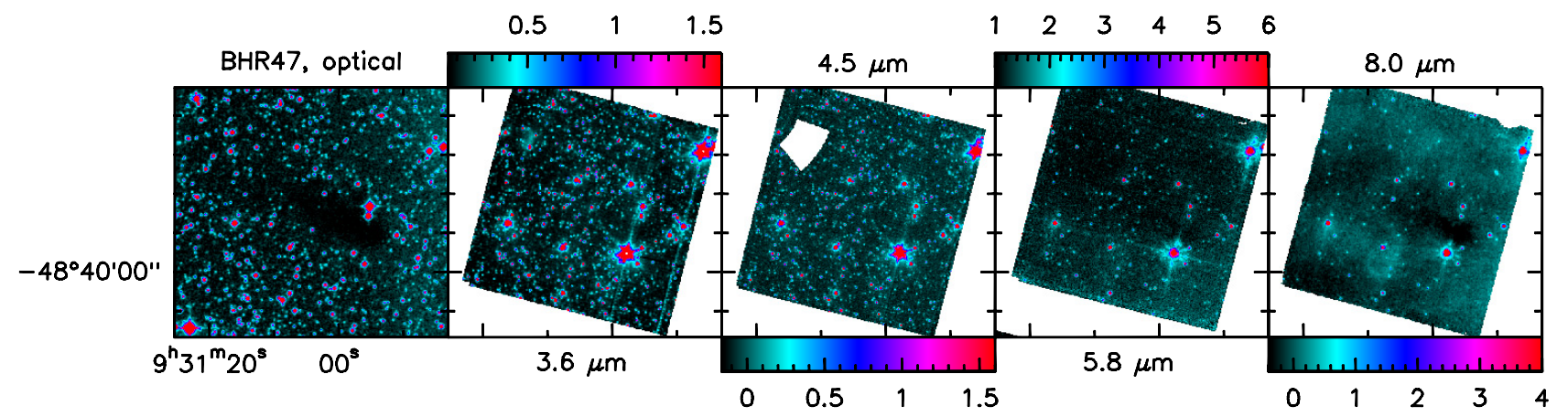

Fig. B.24. Images of BHR47. Absorption. Same as Fig. B.2.

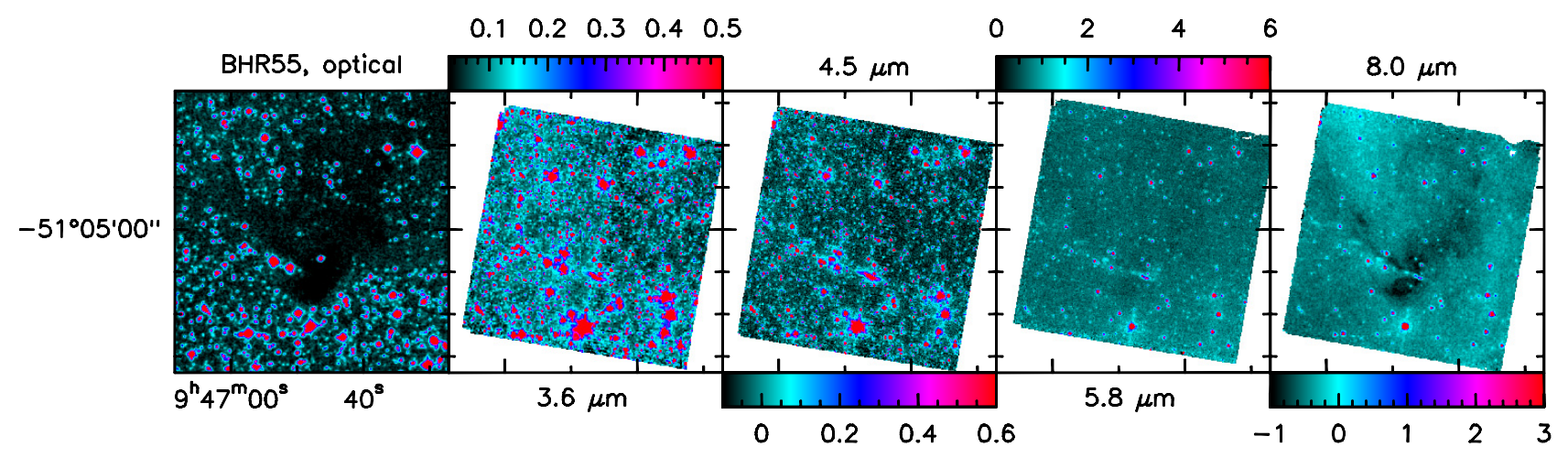

Fig. B.25. Images of BHR55. Absorption, Class 0 object. Same as Fig. B.2.

\section{Appendix C: Optical and Spitzer/IRAC zoomed images of the clouds that show coreshine effect}

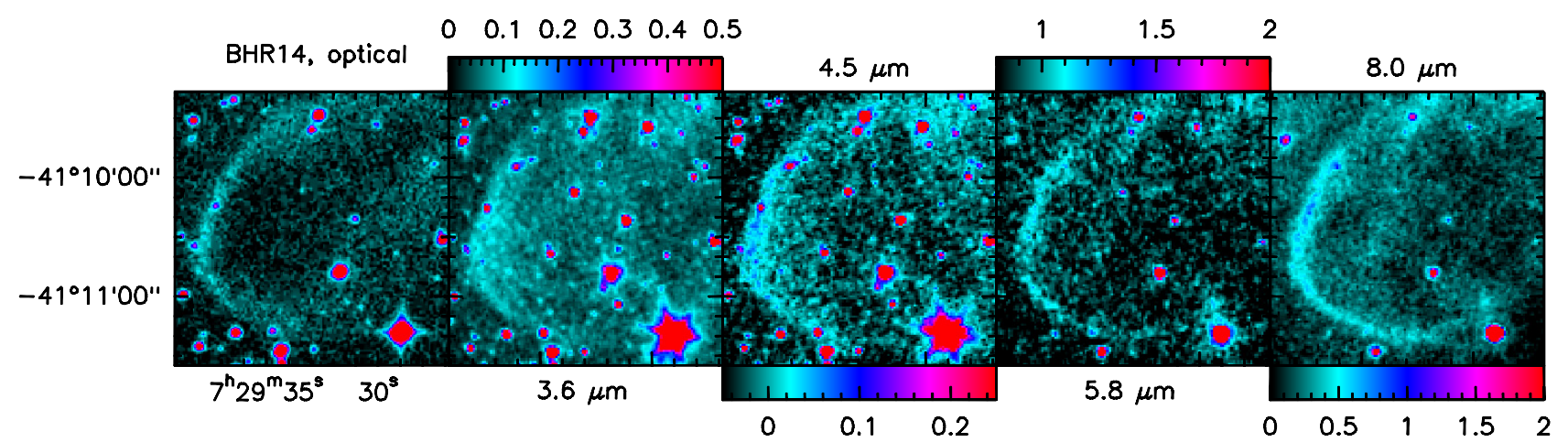

Fig. C.1. Zoom images of BHR14. If real, the coreshine emission fills the CG. The doubt comes from emission at $8 \mu \mathrm{m}$, probably due to PAH emission at the surface of the CG, which masks the expected absorption from the big grains. Same as Fig. B.2.

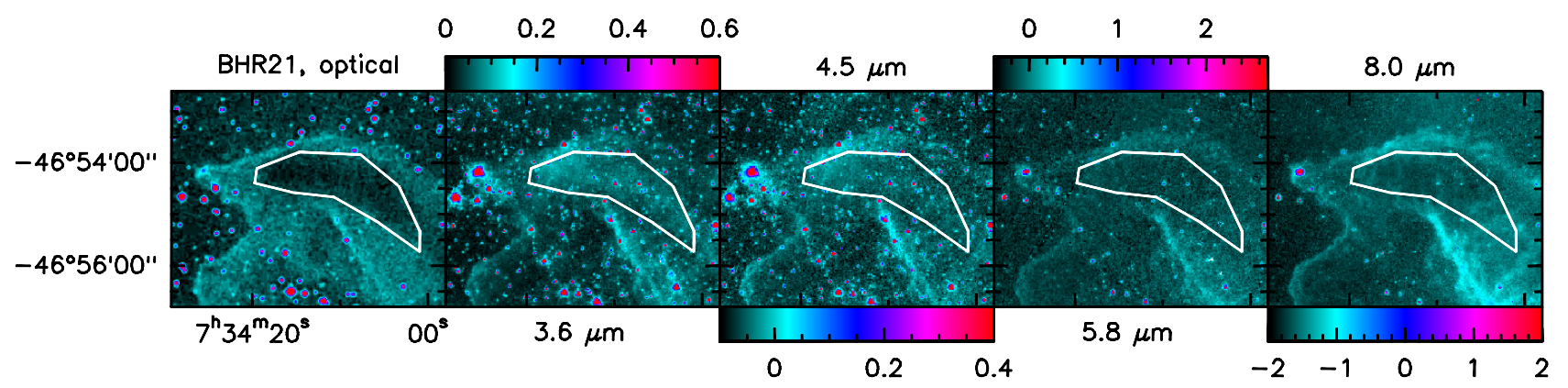

Fig. C.2. Zoom images of BHR21. Possibly the only case of extended coreshine (inside the hand-traced polygon). Same as Fig. B.2. 


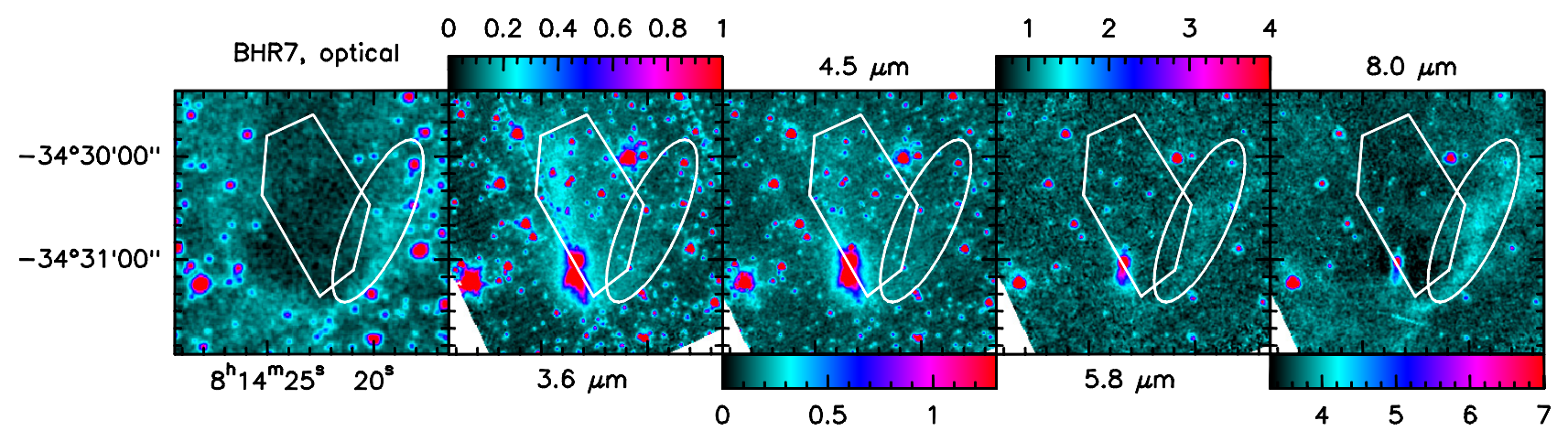

Fig. C.3. Zoom images of BHR7. The ellipse traces the PAH emission region, while the polygone traces the coreshine emission region. This emission seems to benefit from the presence of the protostar extended emission. Same as Fig. B.2.

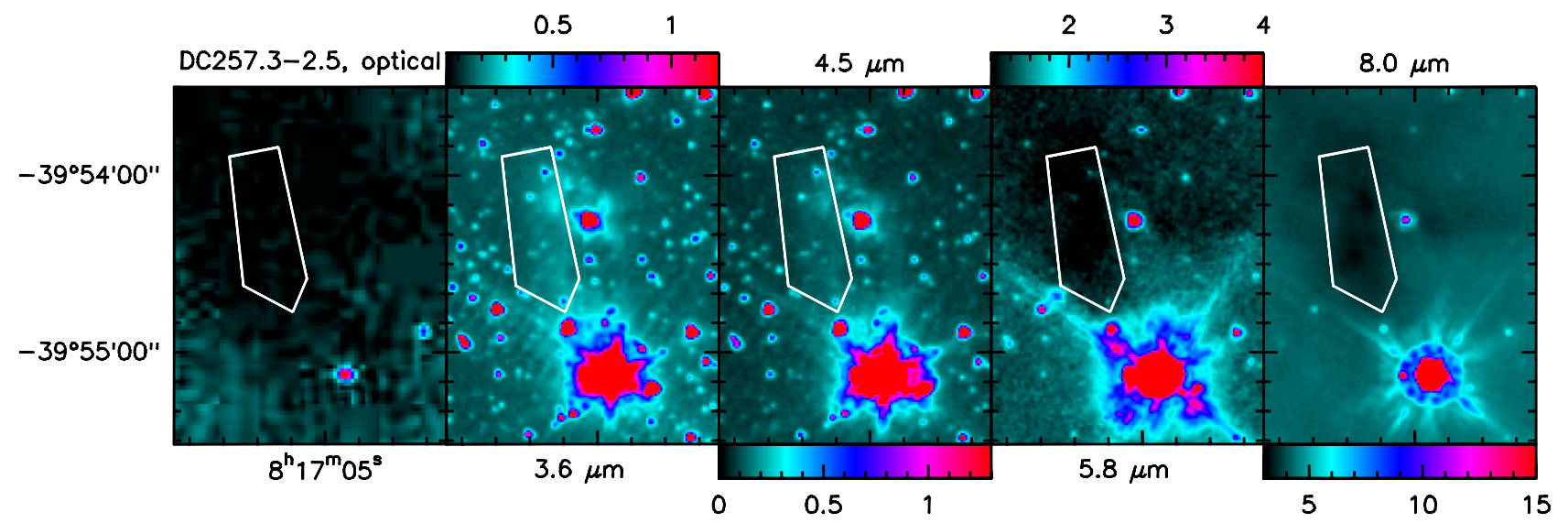

Fig. C.4. Zoom images of DC257.3-2.5. The polygon traces the coreshine emission region. This emission could benefit from the presence of the (probable) protostar situated on its right or of the IRAS source at the bottom, though the link of the IRAS source with the cloud has not been checked yet and is possibly a chance alignment. There is also possibly some coreshine on the righthand side of the (proto)star but it is highly uncertain. Same as Fig. B.2.

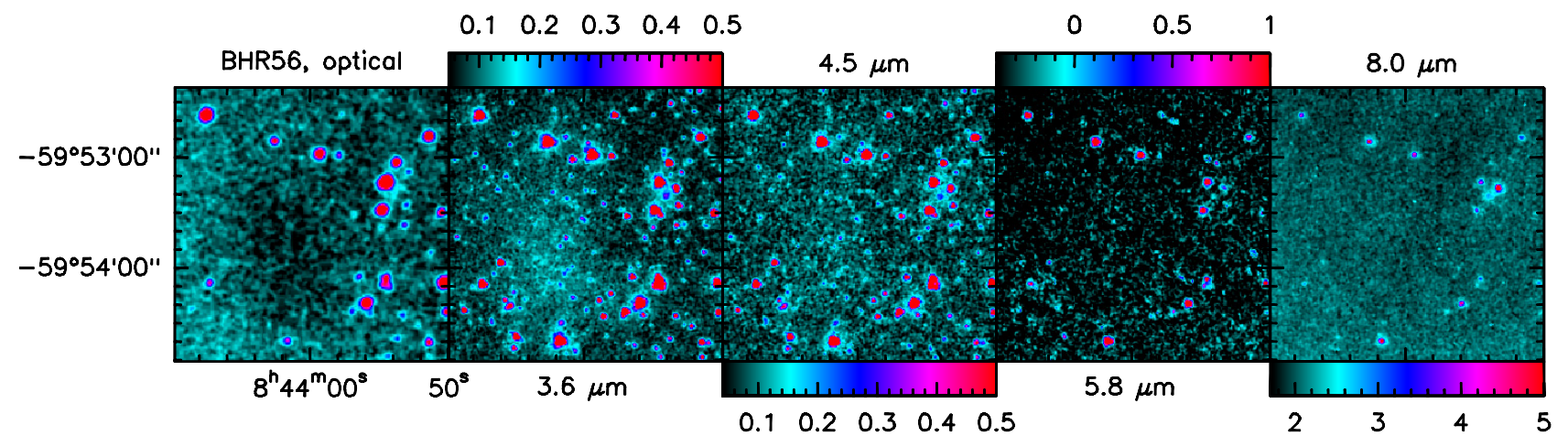

Fig. C.5. Zoom images of BHR56.The coreshine emission is clearly visible by comparing the optical and the $3.6 \mu \mathrm{m}$ images. The 4.5 image hardly shows any enhancement. Absorption is not visible at the longest wavelengths. Such a case of coreshine emission was already witnessed in our previous survey (Pagani et al. 2010). Same as Fig. B.2. 
L. Pagani et al.: Absence of coreshine in the Gum/Vela region $(R N)$

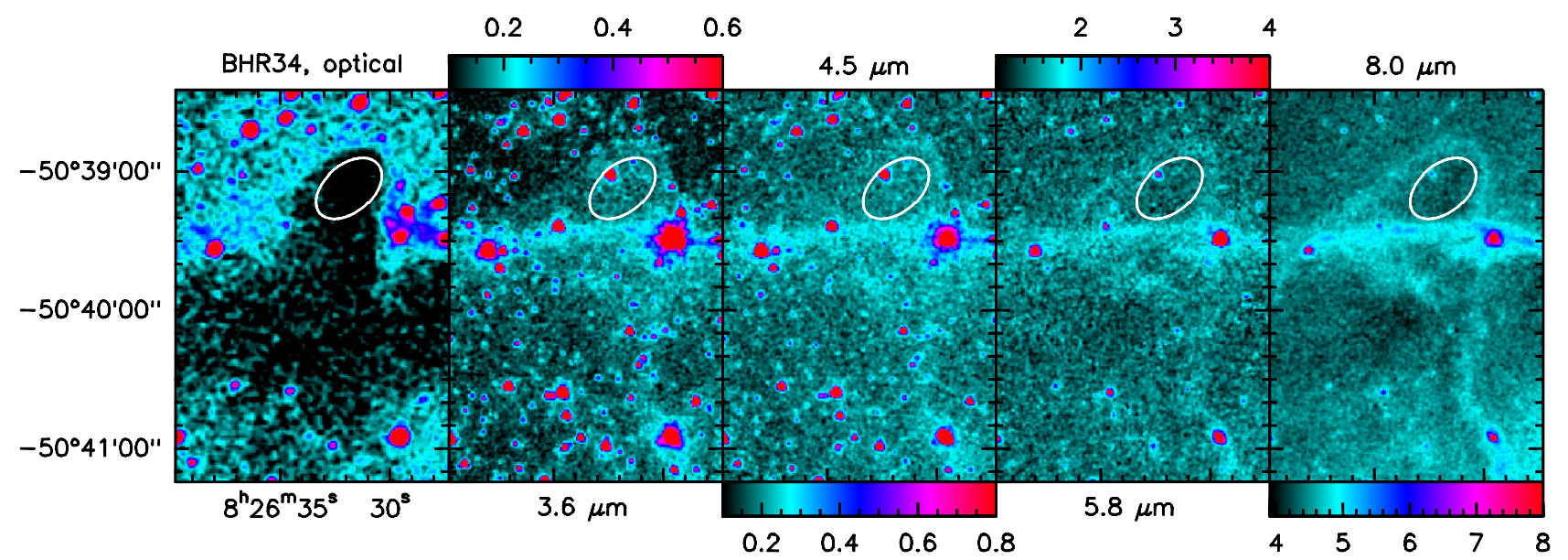

Fig. C.6. Zoom images of BHR34. The ellipse traces the possible coreshine emission region. Same as Fig. B.2.

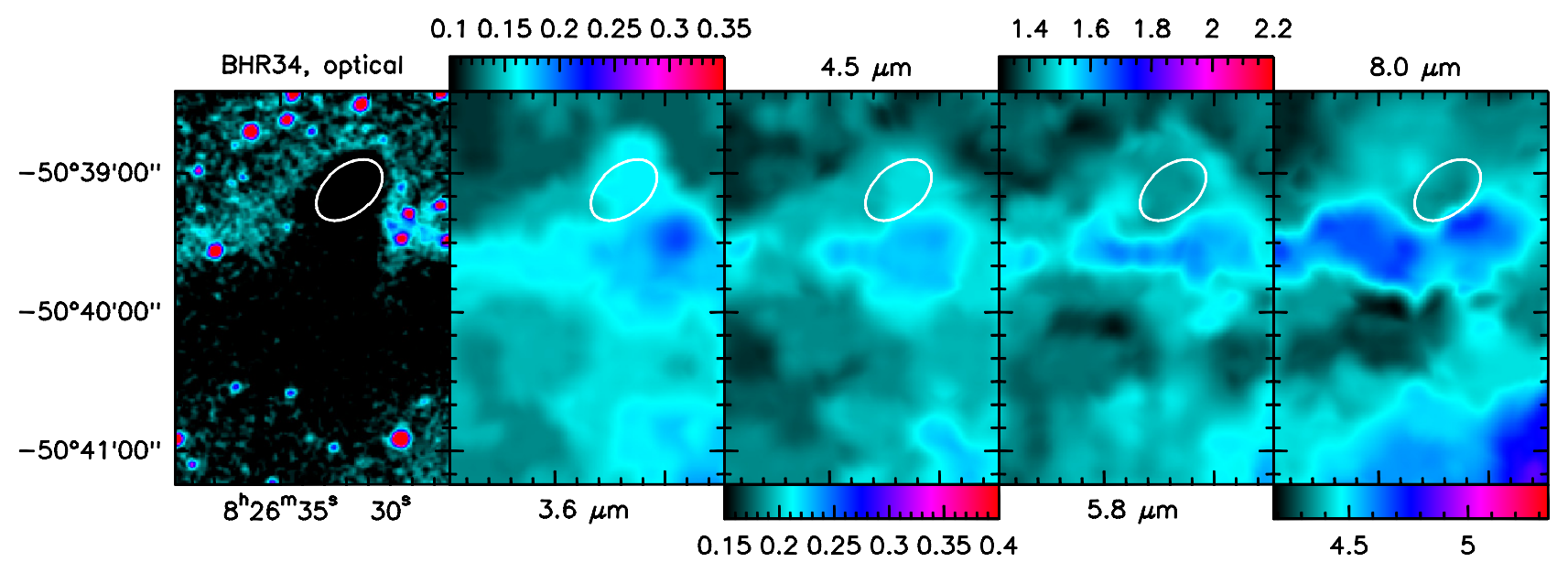

Fig. C.7. Zoom images of BHR34 extended emission in IRAC data (computed with a background estimation function). Coreshine is possibly seen also below the infrared horizontal bar, but is mixed with PAH emission and therefore difficult to establish. Same as Fig. B.2. 